\title{
Dissection of miRNA pathways using Arabidopsis mesophyll protoplasts
}

Cláudia Martinho ${ }^{1 * *}$, Ana Confraria ${ }^{1 *}$, Carlos Alexandre Elias ${ }^{1}$, Pierre Crozet $^{1}$, Ignacio Rubio-Somoza ${ }^{2}$, Detlef Weigel ${ }^{2}$, Elena Baena-González ${ }^{1}$

1 Plant Stress Signaling, Instituto Gulbenkian de Ciência, Rua da Quinta Grande - 6, 2780-156 Oeiras, Portugal

${ }^{2}$ Department of Molecular Biology, Max Planck Institute for Developmental Biology, D-72076 Tübingen, Germany

"These authors contributed equally to this work

₹Present address: Sainsbury Laboratory, Cambridge University, Bateman Street, Cambridge, CB2 1LR, UK

Running title: miRNA pathways in Arabidopsis protoplasts

Short summary: We developed a set of sensors based on the firefly luciferase reporter gene to quantify miRNA activity in vivo, using Arabidopsis mesophyll protoplasts. We show that these sensors are not only responsive to endogenous miRNA activity, but also to exogenous gain- and loss-of-function manipulation of miRNA abundance. As examples, our sensors can be used for studying regulatory mechanisms of miRNA-associated components, for identifying novel factors with miRNA-associated functions, and for assessing the effect of chemicals on miRNA activity, therefore constituting a powerful tool to dissect miRNA pathways.

Corresponding author: Elena Baena-González

Phone: +351214464630

Fax: +351214407970

Email: ebaena@igc.gulbenkian.pt 


\begin{abstract}
microRNAs (miRNAs) control gene expression mostly post-transcriptionally by guiding transcript cleavage and/or translational repression of complementary mRNA targets, thereby regulating developmental processes and stress responses. Despite the remarkable expansion of the field, the mechanisms underlying miRNA activity are not fully understood. In this paper, we describe a transient expression system in Arabidopsis mesophyll protoplasts that is highly amenable for the dissection of miRNA pathways. We show that by transiently overexpressing primary miRNAs and target mimics, we can manipulate miRNA levels and consequently impact on their targets. Furthermore, we developed a set of luciferase-based sensors for quantifying miRNA activity that respond specifically to both endogenous and overexpressed miRNAs and target mimics. We demonstrate that these miRNA sensors can be used to test the impact of putative components of the miRNA pathway on miRNA activity, as well as the impact of specific mutations, either by overexpression or by the use of protoplasts from the corresponding mutants. We further show that our miRNA sensors can be used for investigating the effect of chemicals on miRNA activity. Our cell-based transient expression system is fast and easy to set up and generates quantitative results, being a powerful tool for assaying miRNA activity in vivo.
\end{abstract}

Keywords: miRNA, miRNA activity, miRNA sensor, protoplasts, Arabidopsis thaliana 


\section{Introduction}

microRNAs (miRNAs) are a class of small 19-24 nt RNAs that regulate gene expression mostly post-transcriptionally (Rogers and Chen, 2013). miRNAs are transcribed from nuclear encoded MIR genes into a primary miRNA, which in plants is processed in two sequential steps in the nucleus by Dicer-Like 1 (DCL1) into the final mature miRNA (Kurihara and Watanabe, 2004). The mature miRNA is then exported into the cytoplasm and loaded into the RNAinduced silencing complex (RISC), which contains a member of the Argonaute (AGO) protein family as a main component. Once incorporated into the RISC, one of the miRNA strands is degraded, while the other triggers transcript cleavage and/or translation repression of complementary mRNA targets (Vaucheret et al., 2004).

miRNAs play essential roles in plant developmental processes, such as morphogenesis (Palatnik et al., 2003), patterning (Zhu et al., 2011), polarity establishment (Liu et al., 2009) and phase transitions (Aukerman and Sakai, 2003; Wang et al., 2009; Wang et al., 2008; Wu et al., 2009; Zhu and Helliwell, 2011). A role for miRNAs has also recently emerged as regulators of several stress responses, such as nutrient deprivation (Hsieh et al., 2009; Liang et al., 2012; Pant et al., 2009; Sunkar et al., 2007), biotic and abiotic stress (RuizFerrer and Voinnet, 2009).

Despite the extensive overall conservation of miRNA pathway components, there are relevant differences between the modes of action of plant and animal miRNAs. In plants, miRNAs and their target sites are highly complementary (Brodersen and Voinnet, 2009; Jones-Rhoades and Bartel, 2004; Tang et al., 2003), whereas, in animals, complementarity between miRNAs and target sites is much lower and mostly restricted to the seed region, nucleotides 2-8 from the 5' end of the miRNA (Grimson et al., 2007). In plants, the target sites can be located within the coding sequence or the UTRs, but, in animals, target sites tend to be in the 3'UTR (Gu et al., 2009). Furthermore, despite the increasing recognition of translational repression as an important mode of miRNA action (Brodersen et al., 2008; Lanet et al., 2009; Li et al., 2013b), transcript cleavage is generally considered the main mechanism in 
plants, whilst in animals translation repression is predominant (Ameres and Zamore, 2013).

The broad impact of miRNAs is dependent on the number and diversity of targets they regulate, as well as on the mechanisms of target regulation. So far, around 120 miRNA targets have been validated in plants (Folkes et al., 2012), but a combination of several bioinformatic prediction tools can yield a list of nearly 5000 predicted targets for the $\sim 300$ mature miRNAs of Arabidopsis thaliana (Confraria et al., 2013).

In addition to understanding the functional interactions between miRNAs and potential targets, a complete comprehension of miRNA function requires knowledge on how miRNAs are regulated and act on their targets. Mature miRNA levels often correlate with changes in miRNA activity (Brodersen and Voinnet, 2009; Jones-Rhoades et al., 2006). Accordingly, northern blotting, quantitative real-time PCR (qRT-PCR), microarrays and deep sequencing have been extensively used to quantify endogenous miRNAs and, thereby, infer changes in their activity (Hsieh et al., 2009; Liu et al., 2008; Lv et al., 2010; Moxon et al., 2008a; Navarro et al., 2006). miRNA levels may be regulated at multiple steps during their biogenesis, such as MIR gene expression (Cho et al., 2012; Hajdarpasic and Ruggenthaler, 2012; Jeong et al., 2011; Liang et al., 2012; Liu et al., 2008; Zhang and Li, 2013), processing of miRNA precursors (Jung et al., 2012; Yan et al., 2012) or miRNA stability (Ramachandran and Chen, 2008). However, studies in animals indicate that the miRNA pathway is extensively regulated also downstream of miRNA biogenesis (Leung and Sharp, 2007), and that the extent of target repression cannot be entirely predicted from the abundance of its regulatory miRNA (Brown et al., 2007). Mechanisms for modulating miRNA activity independently of mature miRNA accumulation have also been described in plants, including different AGO1 subcellular localization, regulation of miRNA loading and/or RISC assembly and miRNA accessibility (Brodersen et al., 2012; Iki et al., 2012; Wang et al., 2011; Zhu et al., 2011). For example, it has been reported that, despite similar miR159 levels, miR159 activity is attenuated in the seed compared to vegetative tissues (Alonso-Peral et al., 2012). Altogether, these findings indicate that miRNA activity is 
determined by factors other than miRNA abundance and therefore additional methods that do not rely on miRNA quantification are required to monitor miRNA activity.

Alterations in the transcriptional profile of known and predicted miRNA targets have been previously used to assess changes in miRNA activity in different conditions or genotypes (Confraria et al., 2013; Laubinger et al., 2010; Moldovan et al., 2010; Ronemus et al., 2006; Todesco et al., 2010). However, this approach by itself does not allow uncoupling miRNA-mediated suppression from additional regulatory mechanisms acting on miRNA targets, neither does it consider the effects of translational attenuation.

Alternatively, miRNA activity can be quantified using "miRNA sensors", reporter genes harboring one or more copies of miRNA sensitive sequences. In animals, luciferase-derived reporters have been widely used in cell-based assays to quantify miRNA activity and have underpinned key studies on miRNA biology (Beillard et al., 2012; Brown et al., 2007; Connelly et al., 2012; Doench and Sharp, 2004; Gibbings et al., 2012; Horman et al., 2013; Huang et al., 2009; Humphreys et al., 2005; Janas et al., 2012; Lytle et al., 2007; Mullokandov et al., 2012; Petersen et al., 2006). Additionally, luciferase-based reporter systems are extensively used to validate miRNA targets in animal cells (Nicolas, 2011). On the other hand, in plants, stable lines expressing GFP- or DsRed-based small RNA sensors have been previously generated to assess miRNA activity (Carlsbecker et al., 2010; Marin et al., 2010; Nodine and Bartel, 2010; Schwab et al., 2009). Transient transformation of Nicotiana benthamiana leaves with a miRNA GFP reporter was also reported (Parizotto et al., 2004). These strategies often involve time-consuming stable plant transformation and/or lack quantitative outputs. The recognition of such drawbacks has prompted the development of alternative methods for monitoring plant miRNA activity that rely on transient expression systems and luciferase reporters. Iwakawa and Tomari (Iwakawa and Tomari, 2013) developed an in vitro system with extracts from cultured plant cells, which they used to explore the mechanisms regulating miRNA-induced translation repression, whereas Liu and 
colleagues (Liu et al., 2014) used Nicotiana benthamiana to investigate the complementarity requirements between miRNAs and their targets.

We describe here a method for quantifying miRNA in vivo using luciferase-based miRNA sensors in Arabidopsis mesophyll protoplasts. Transient expression of miRNA sensitive and resistant forms of these sensors in Arabidopsis protoplasts enables fast and specific quantification of endogenous miRNA activity. Overexpression of miRNAs and target mimics in Arabidopsis protoplasts not only impacts the levels of endogenous targets, but also the levels of co-expressed reporters, allowing the manipulation of system at multiple levels. The protoplast-miRNA reporter system can be readily applied to investigate the biology of miRNA machinery components and mutants deficient in miRNA function, as well as to address the impact of specific compounds or treatments, thereby establishing a highly versatile, robust, and easy method to dissect miRNA pathways in plants.

\section{Results}

\section{Transient miRNA loss and gain-of-function in Arabidopsis protoplasts}

To evaluate whether Arabidopsis protoplasts are an amenable system for miRNA research we first tested if manipulating miRNA levels would impact on the endogenous targets. We used a combined gain and loss-of-function strategy overexpressing miRNAs and target mimics, respectively. We overexpressed specific miRNAs by transfecting protoplasts with a plasmid harboring the potential primary miRNAs (Cuperus et al., 2010; Schwab et al., 2006) under the control of the constitutive $35 S$ promoter. As shown in Figure $1 \mathrm{~A}$, this sequence is sufficient for the correct processing and accumulation of the corresponding mature miRNAs, even in the case of miR161, in which two mature miRNAs are produced from the same primary transcript (Figure S1). To assess if mature miRNA accumulation triggered mRNA target repression, we performed qRT-PCR for MYB33/MYB65 and TCP2/TCP4, well-established targets of miR159 and miR319, respectively (Achard et al., 2004; Millar and Gubler, 2005; Palatnik et al., 2003; Palatnik et al., 2007; Schwab et al., 2005). 
These two miRNAs share very similar sequences, and although miR159 does not affect TCP2/TCP4, miR319 can direct the cleavage of MYB33 and MYB65 (Palatnik et al., 2007). Overexpression of miR319 in protoplasts caused a $70 \%$ reduction in TCP2 and $82 \%$ in TCP4 levels, and this decrease was specific, since TCP2 and TCP4 levels were not reduced upon miR159 or miR161.1/2 overexpression (Figure 1B). Curiously, miR161.1/2 overexpression resulted in an increase in TCP2 but not in TCP4 levels, suggesting a miR319-independent effect potentially related to $T C P 2$ transcription or stability. In the case of the miR159 targets MYB33 and MYB65, the repression by miR159 overexpression was not statistically significant, even though the impact of miR159 was stronger than that of miR319 or miR161.1/2 (Figure 1C). The differential effect of miR319 and miR159 overexpression on their corresponding targets is probably due to differences in the endogenous levels of these two miRNAs [Figure 1A; (Kozomara and Griffiths-Jones, 2011)]. miR319 levels are rather low in Arabidopsis protoplasts and consequently miR319 overexpression has a strong effect on its target transcripts (Figure 1B), whilst miR159 is highly abundant, being more difficult to further enhance its activity by overexpression (Figure 1C).

In parallel to miRNA overexpression, we overexpressed target mimics as a way to downregulate specific miRNAs (Figure 2A). Target mimicry is the term coined to designate the sequestration of a miRNA by an RNA molecule that is only partly complementary to the miRNA, creating a bulge in the area where cleavage would occur in case of functional complementarity (Franco-Zorrilla et al., 2007; Todesco et al., 2010). This prevents miRNA function and targets miRNAs for degradation (Todesco et al., 2010). Indeed, transient overexpression of target mimics for miR159 (MIM159), miR161.1 (MIM161.1) and miR161.2 (MIM161.2) was sufficient to decrease the levels of the corresponding miRNAs in protoplasts (Figure 2A). The low abundance of miR319 precluded its detection in small RNA blots both in control cells and in cells overexpressing a miR319 target mimic (MIM319; not shown). Nevertheless, MIM319 overexpression increased TCP4 accumulation (Figure 2B). Even though TCP2 was not significantly increased by MIM319 overexpression, it accumulated to a similar extent as in transgenic plants 
overexpressing MIM319 (Franco-Zorrilla et al., 2007; Todesco et al., 2010) (Figure S2), showing that transient expression of MIM319 in protoplasts is comparable to its stable transformation into plants. On the other hand, miR159 targets MYB33 and MYB65 were on average 10 times up-regulated by the expression of a miR159 target mimic (MIM159; Figure 2C). As for miRNA overexpression, the mild effect of MIM319 on TCPS compared to the strong impact of MIM159 on MYBs likely reflects differences in the endogenous levels of these two miRNAs: sequestering a lowly abundant miRNA such as miR319 impacts mildly on its targets, whilst sequestering a highly abundant miRNA such as miR159 has a strong impact on its targets. In addition, MIM159 overexpression induced accumulation of TCP4 and a mild but not significant increase in TCP2 levels (Figure 2B). MIM319 overexpression, in turn, induced also the accumulation of MYB33/MYB65 (Figure 2C), in agreement with what has been reported in plants overexpressing MIM319 (Franco-Zorrilla et al., 2007). This probably occurs because of the high similarity of these two miRNAs and the partial sequestration of miR319 and miR159, respectively. Supporting this, the unrelated target mimics MIM161.1 and MIM161.2 had a negligible effect on TCP2/TCP4, as well as on MYB33/MYB65 (Figures 2B, 2C).

One of the most commonly used strategies to identify and validate miRNA targets in the animal field is to transiently overexpress miRNAs and evaluate the effects on potential targets, either at the mRNA or protein level (Lim et al., 2005; Selbach et al., 2008). However, miRNA overexpression may induce off-target effects (Burchard et al., 2009; Khan et al., 2009) and is therefore often combined with a miRNA loss-of-function approach (Baek et al., 2008; Krutzfeldt et al., 2005; Selbach et al., 2008). As described above, such a combined strategy can be easily adapted for Arabidopsis protoplasts. So, we tested if PPDK (AT4G15530), a predicted target of miR159, and ALDHL22A1 (AT3G66658), a predicted target of miR319 (Allen et al., 2005; Dai and Zhao, 2011; Fahlgren et al., 2007; Moxon et al., 2008b) were indeed true, functional targets of these miRNAs. However, neither overexpression of miR159 or miR319 nor overexpression of the corresponding target mimics caused any significant changes on PPDK and ALDH22A1 mRNA levels (Figures S3, S4), 
excluding these transcripts as miR159 and miR319 targets, respectively, and ruling out possible off-target effects of miRNA or MIM overexpression. Using a different approach of target and miRNA co-expression and western blotting to monitor target expression, a recent study also ruled out $A L D H 22 A 1$ as a miR319 target (Li et al., 2013a).

\section{Use of miRNA sensors for quantifying miRNA activity}

To quantify miRNA activity without measuring mature miRNA levels, we developed a miRNA sensor system that employs firefly luciferase ( $f L U C$ ) as a reporter gene in transient protoplast assays, providing normalized luciferase activity as an inverse quantitative readout of miRNA activity. The miRNA sensors were generated by introducing a specific miRNA binding site corresponding to a known target in the 3'UTR of the fLUC transcript (Figures 3, S5). The miRNA target site is expected to pair with the endogenous miRNA, which will thereby mediate its repression by mRNA cleavage and/or translational repression. As examples, we developed sensors to quantify specifically miR319, miR159, and miR161.2 activities using the target sequences of TCP4, MYB33 (Figure 3), and AT1G61350 (Figure S5), respectively. These sensors are hereafter referred as $f L U C_{T C P 4}, f L U C_{M Y B 33}$, and fLUC $_{A T 1 G 63150 .}$ MYB33 and AT1G63150 were selected based on their lowest free energy from all the possible validated miRNA-target pairs for miR159 and miR161.2, respectively [http://wmd3.weigelworld.org/cgi-bin/webapp.cgi; (Allen et al., 2004; Schwab et al., 2006)]. Interestingly, MYB33 has the lowest free energy for miR319 as well, and has been shown to be an occasional target of miR319 in wild-type (WT) plants (Palatnik et al., 2007). However, to generate a miR319 sensor we selected the TCP4 target sequence as it is an established target of this miRNA for which the hybridization energy is also low (Figure 3). To simplify, as these sensors are expected to undergo miRNA-mediated cleavage

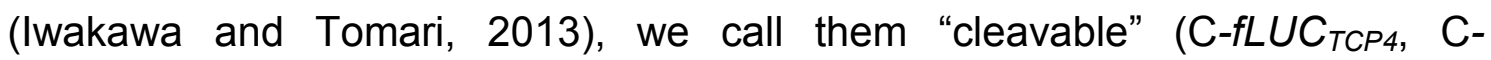

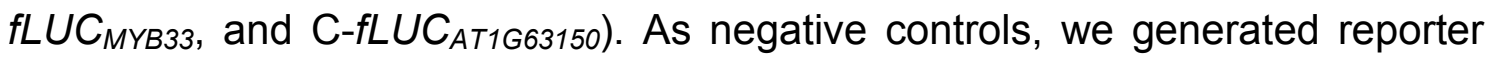
variants bearing two mutations on the positions complementary to 10 and 11 of the corresponding miRNAs, and designated these as "non-cleavable" (NC- 
$f L U C_{T C P 4}, N C-f L U C_{M Y B 33}$, and $N C-f L U C_{A T 1663150)}$, as mutations in these sites have been shown to be important for slicing (Llave et al., 2002; Parizotto et al., 2004). For the miR161.2 sensor, we generated an additional control with multiple mutations in the seed region (Figure S5), which behaved essentially as the non-cleavable version (Figure S6).

When expressed in Arabidopsis protoplasts, luciferase activity of the cleavable $f L U C_{M Y B 33}$ was significantly lower than that of its non-cleavable counterpart (Figure 4A), supporting the occurrence of high miR159 activity in protoplasts and the sensitivity of the cleavable $f L U C_{M Y B з 3}$ reporter to endogenous miR159 action. Furthermore, expression of MIM159 reverted cleavable $f L U C_{M Y B 33}$ activity to levels similar to those of non-cleavable $f L U C_{M Y B 33}$ (Figure $4 \mathrm{~A}$ ), on which it had no effect. On the other hand, luciferase activity was similar in both cleavable and non-cleavable versions of the miR319 sensor $f L U C_{T C P 4}$ and MIM319 expression had no effect on the cleavable $f L U C_{T C P 4}$ reporter, or on its non-cleavable variant (Figure 4B). Presumably, this is again a reflection of the low basal activity of miR319 in Arabidopsis protoplasts and is in agreement with our previous observations on the mild impact of MIM319 on the endogenous miR319 targets TCP4 and TCP2 (Figure $2 B$ ). Altogether, these results show that luciferase activity measured from the miRNA sensors can be used as a proxy for the activity of the corresponding endogenous miRNAs.

To further test the ability of the sensors to detect changes in miRNA activity, we co-expressed them together with the corresponding miRNAs. Surprisingly, overexpression of miR159 and miR319 failed to repress the cleavable $f L U C_{M Y B 33}$ and $f L U C_{T C P 4}$ reporters, respectively. Given that the miRNA reporters are expressed from the strong $35 \mathrm{~S}$ promoter (Figure 3), triggering an over-accumulation of the miRNA-binding target sites, we hypothesized that the lack of reporter repression upon miRNA overexpression could be due to limiting AGO activity in Arabidopsis protoplasts. Since AGO1 is the main slicing effector of the miRNA-loaded RISC complex (Mallory et al., 2008), we co-expressed AGO1 with each one of the miRNAs, which together repressed the cleavable reporters $f L U C_{M Y B 33}$ and $f L U C_{T C P 4}$, by $72 \%$ and $87 \%$, 
respectively, but had no impact on the corresponding non-cleavable versions (Figures 4A, 4B). AGO1 alone had a mild effect on the cleavable reporters, in particular on $f L U C_{T C P 4}$, but its effect was dramatically enhanced by the coexpression of the respective miRNAs. Interestingly, the expression of AGO1 alone, but not of AGO1 and miRNA, also decreased the activity of the noncleavable $f L U C_{M Y B 33}$. In this particular case, in which the there is no central complementarity and the stoichiometry of the miRNA:target pair is shifted towards the target, overexpressed AGO1 "guided" by endogenous miR159 may trigger a non-cleavage repression mechanism, presumably translation repression, similarly to the effect observed in a recent study on the miR159MYB33/MYB65 regulatory module ( $\mathrm{Li}$ et al., 2014). This effect is abolished when AGO1 is co-expressed with the primary miRNA, possibly because the stoichiometry of miRNA:target pair becomes again more balanced. On the noncleavable $f L U C_{T C P 4}$, the effect of $A G O 1$ alone is not visible, which may be yet another reflection of the difference in abundance between the two miRNAs.

We next asked whether reporter repression induced by $A G O 1$ and miRNA co-expression was due to transcript cleavage or to translation inhibition. To this end, we quantified LUC mRNA using qRT-PCR and primers flanking the miRNA binding site. These analyses revealed that co-expression of AGO1 and miRNA induced a $65 \%$ and $80 \%$ reduction in the expression of the cleavable $f L U C_{M Y B 33}$ and $f L U C_{T C P 4}$ reporters, respectively, whilst having no significant impact on their non-cleavable counterparts (Figures 4C, 4D). Given that coexpression of AGO1 and miRNA caused a similar reduction in LUC activity ( $72 \%$ and $87 \%$ for $f L U C_{M Y B 33}$ and $f L U C_{T C P 4}$, respectively, Figures $4 A, 4 B$ ), these results indicate that reporter repression occurs mostly through transcript cleavage. Altogether, our results show that the sensors respond specifically to miRNA action. Furthermore, the data suggests that $A G O 1$ activity is limiting when the target and the miRNA are co-expressed and that AGO1 and miRNA act together mainly by cleaving the LUC transcript. 


\section{miRNA sensors are highly specific}

In order to assess the specificity of the miRNA sensors, we co-expressed the cleavable reporters and AGO1 together with different miRNAs (Figures 4E, 4F). The $f L U C_{M Y B 33}$ reporter was significantly repressed by AGO1 and miR159 coexpression (Figure 4E). AGO1 and miR319 co-expression also reduced $f L U C_{M Y B 33}$ activity by $35 \%$, which is consistent with the impact of miR319 on endogenous MYB33 (Figure 1C) and with the observation that MYB33 cleavage products are more abundant in 35S:::miR319a overexpressor plants than in WT (Palatnik et al., 2007). Similarly to what happened in the case of the endogenous targets, MIM319 was able to partially revert the effect of miR159 and $A G O 1$ on $f L U C_{M Y B 33}$, although this effect was clearly lower than that of the more specific MIM159 (Figure 4E). In parallel, co-expression of AGO1 with miR319 strongly repressed the $f L U C_{T C P 4}$ reporter whereas co-expression with miR159 had no effect (Figure 4F), showing that this sensor responds specifically to higher miR319 accumulation. In addition, miR319-mediated repression could be reverted to control levels by co-expression with MIM319 whereas MIM159 had no clear effect (Figure 4F). Additionally, the sequenceunrelated target mimic for miR161.2 (MIM161.2) could not revert the effects of the combination of AGO1 with either miR159 or miR319 on their respective reporters, $f L U C_{M Y B 33}$ and $f L U C_{T C P 4}$ (Figures S7A, S7B). Likewise, the repression of $A G O 1$ and pri161 on the miR161.2 reporter $f L U C_{A T 1 G 63150}$ could be reverted by MIM161.2, but not by MIM161.1, MIM159 or MIM319 (Figure S7C), confirming the specificity of our reporters.

\section{miRNA sensors are valuable tools to study the miRNA pathway}

Overexpressed miRNAs require the simultaneous expression of AGO1 for efficiently repressing miRNA sensors (Figure 4). Hence, this assay may be applied to investigate AGO1 regulation and action, for example, by using mutated forms of AGO1 or by co-expressing additional components with a potential effect on AGO1 function. To demonstrate this, we generated AGO1 variants impaired in mRNA slicing by mutating two conserved residues of the catalytic site (D760A and D848A) (Baumberger and Baulcombe, 2005; 
Carbonell et al., 2012) and tested their effect on the miRNA reporters (Figures $5 \mathrm{~A}, 5 \mathrm{~B})$. For both $f L U C_{M Y B 33}$ and $f L U C_{T C P 4}$ reporters, a reduction of luciferase activity was only observed when the corresponding miRNA was co-expressed with WT AGO1 but not with catalytically dead $A G O 1^{D 760 A}$ or $A G O 1^{D 848 A}$. The lack of repression was not due to impaired protein accumulation, as the mutated AGO1 variants accumulated in protoplasts to much higher levels than WT AGO1 (Figures 5A, 5B). This in turn suggests that AGO1 stability is inversely correlated with its activity. Furthermore, our data are consistent with the previously described inability of these AGO1 mutants to carry out mRNA slicing (Baumberger and Baulcombe, 2005; Carbonell et al., 2012), and suggest that reporter repression upon miRNA overexpression is mainly due to mRNA cleavage rather than translation attenuation. This is in agreement with the conclusions drawn from the relative quantification of LUC expression by qRTPCR (Figures 4C, 4D), but also with the conclusions from $\mathrm{Li}$ and colleagues ( $\mathrm{Li}$ et al., 2014), who showed that perfect central complementarity and a high miRNA:target ratio promote cleavage.

To test the system further, we asked whether our protoplast reporter system could be used for assessing the effect of specific compounds on miRNA activity. As a proof of concept, we selected two well-established inhibitors of HSP90, geldanamycin and radicicol, since AGO1 interacts with HSP90 and this interaction is important for the assembly of a functional RISC complex both in humans and plants (Iki et al., 2010; Iwasaki et al., 2010; Miyoshi et al., 2010). We further selected the cysteine protease inhibitor E64d, which blocks protein degradation through autophagy (Asanuma et al., 2003). The rationale behind this choice was that AGO1 and AGO2 are degraded by autophagy in Arabidopsis and humans and AGO2 degradation through autophagy was shown to be required for sustaining miRNA activity (Derrien et al., 2012; Gibbings et al., 2012). As shown in Figures $5 C$ and $5 D$, the repression of the $C-f L U C_{M Y B 33}$ and $\mathrm{C}-\mathrm{fLUC} \mathrm{C}_{\mathrm{TCP} 4}$ reporters caused by $\mathrm{AGO1}$ and miRNA co-expression was completely blocked by radicicol and to a lower extent by geldanamycin, supporting the importance of HSP90 for RISC function. No effect was however 
observed for E64d, suggesting that in Arabidopsis or at least in our system, autophagy is not essential for sustaining miRNA-mediated cleavage.

Altogether, these results show that our reporter assays allow not only functional studies of AGO1 and possibly of other components of the miRNA pathway, but also can be used to explore the effect of chemicals on miRNA activity.

To further test the applicability of our system for the functional dissection of the miRNA pathway we monitored miRNA activity using $f L U C_{\mathrm{TCP} 4}$ and $f L U C_{\mathrm{MYB} 33}$ in protoplasts isolated both from WT and dcl1-9 mutant plants, which are compromised in miRNA processing, and consequently accumulate reduced levels of many miRNAs (Park et al., 2002; Vazquez et al., 2004). The basal activity of both cleavable reporters was higher in dc/1-9 than in WT (Figure 6), reflecting the impaired or reduced accumulation of miRNAs in this mutant. However, for $f L U C_{T C P 4}$, differences in luciferase activity between $d c / 1-9$ and WT were significantly milder than the differences observed with $f L U C_{M Y B 33}$, again reflecting the fact that in the WT, the levels of miR159 are higher than those of miR319. In the case of $f L U C_{M Y B 33}$, the basal activity in $d c / 1-9$ was comparable to the values obtained for MIM159 overexpression in WT (Figure S8A), suggesting that miR159 knock-down using the target mimicry approach reduces miR159 activity to the same extent as it is in dc/1-9. The basal activity of cleavable $f L U C_{M Y B 33}$ in $d c / 1-9$ was also similar to the activity of the non-cleavable $f L U C_{M Y B 33}$ in the WT, further demonstrating the low miR159 activity of the mutant (Figure S8A). For $f L U C_{T C P 4}$, both the cleavable and non-cleavable reporters show similar activity in both WT and $d c / 1-9$ (Figure S8B), consistent with the low basal miR319 activity observed in the WT.

As shown before, miRNA overexpression combined with AGO1 strongly decreased luciferase activity in the WT (Figures 4, 5). However, luciferase activity was significantly higher in $d c / 1-9$ than in the WT (Figure 6), likely reflecting the lack of primary miRNA conversion into mature miRNA in the dc/19 mutant. Importantly, the dc/1-9 mutant could be complemented by coexpression of DCL1, restoring to WT levels the effect of AGO1 and miRNA overexpression on both reporters (Figure 6). 
Finally, we asked whether miRNA reporter repression is exclusively due to direct miRNA action or whether secondary siRNAs (Axtell, 2013) are generated upon overexpression of the reporter, contributing to its repression. To this end, we monitored LUC activity using the cleavable $f L U C_{M Y B 33}$ and $\mathrm{fLUC}_{T C P 4}$ reporters in protoplasts isolated from WT, dc/2-1 (Xie et al., 2004), dc/4-2 (Xie et al., 2005), and rdr6-15 (Allen et al., 2004) plants. Co-expression of AGO1 and miRNA induced equal repression of the reporters in all the tested genotypes (Figure S9), indicating that the observed activities are due to the joined action of the co-expressed AGO1 and miRNA and not to the formation of secondary siRNAs.

In summary, this data further supports the sensitivity of our miRNA sensors to the levels of specific miRNAs, and validates our strategy for functional assays of miRNA pathway components.

\section{Discussion}

Here we propose a rapid, easy, and amenable system for quantification of miRNA activity in vivo by using transient overexpression of miRNA-related components in Arabidopsis protoplasts.

We demonstrated that by combining specific gain- and loss-of-function approaches it is possible to manipulate miRNA levels impacting on endogenous targets. We overexpressed specific miRNAs and the respective target mimics, followed by qRT-PCR to assess differences in the levels of endogenous mRNA targets, characterizing in this way the effect of the miRNA in the physiological context of the target. We used as examples the highly abundant miR159 and the scarce miR319, which are known to target the MYB and TCP transcription factors, respectively. Our results show that miRNA overexpression is more effective for low activity miRNAs, such as miR319, than for high activity miRNAs, such as miR159 (Figure 1). On the other hand, target mimic expression is particularly effective in blocking the activity of the highly abundant miRNAs, as miR159, but has negligible effects on lowly abundant miRNAs, as miR319 (Figure 2). This strategy may be used to validate miRNA-target pairs, similarly to what is usually done in animal studies (Baek et al., 2008; Krutzfeldt 
et al., 2005; Lim et al., 2005; Selbach et al., 2008). It shows the advantage of being possibly adapted to all miRNAs or miRNA candidates and easily coupled to large scale non-biased approaches, such as microarrays. Additionally, it allows the validation of targets of miRNA-guided translation arrest if there are antibodies available for the predicted targets of interest, if miRNAs are coexpressed with tagged versions of the predicted targets, or if a global proteomics analysis is carried out. In fact, miRNA and target co-expression in Arabidopsis protoplasts was independently proposed as a way to validate miRNA targets recently ( $\mathrm{Li}$ et al., 2013a). However, Li and collaborators coexpressed the miRNA with tagged versions of the respective targets and assessed differences by western blotting, whereas we show that in most cases differences can be directly assessed on the transcript levels of endogenous targets, with no need to overexpress the target itself.

Foremost, we present miRNA sensors that allow the quantification of endogenous miRNA activities present in mesophyll cells. Using firefly luciferase as a reporter gene, we introduced in its 3'UTR a miRNA target site or a mutated target site that is resistant to miRNA-guided repression. Our results show that miRNA sensors readily detect the activity of specific endogenous miRNAs. Additionally, our miRNA sensors respond to changes in miRNA levels provoked by miRNA or target mimic overexpression, a property that can be used to evaluate the impact of putative components of the miRNA pathway or of specific mutations in known components. Seeing that efficient repression of the miRNA sensors by overexpression of the corresponding primary miRNAs requires coexpression of $A G 01$, it appears that AGO1 activity is limiting in protoplasts when both the miRNA and the target are expressed to high levels (Figures 4A, $4 \mathrm{~B})$. This contrasts with the results of a recent study employing transient coexpression of targets and artificial miRNAs (amiRs) also in Arabidopsis mesophyll protoplasts, in which repression by amiRs could not be further enhanced by co-expression with AGO1, AGO2, AGO4 or AGO10 ( $\mathrm{Li}$ et al., 2013a). amiRs are designed to harbor a uridine at position 1, which, in endogenous miRNAs, is known to allow their preferential association with AGO1 (Mi et al., 2008). However, in spite of their high efficiency, amiRs were 
not shown to be associated with AGO1 or any of the AGOs tested by Li and colleagues. As we reproducibly see that it is necessary to co-express AGO1 for effective repression of the cleavable sensors by overexpressed miRNAs, we can only postulate that the mechanisms of regulation differ between miRNA and amiR-mediated repression. amiR-mediated regulation was found to be exerted mainly through translational repression ( $\mathrm{Li}$ et al., 2013a), a process in which AG010 plays an important role (Brodersen et al., 2008). It is hence possible that amiRs are mostly loaded into AGO10, and that AGO10 activity is not limiting in protoplasts.

miRNA sensors can be easily adapted to study the activity of other miRNAs, and provide an easy and highly versatile system to carry out functional studies of miRNA pathway components in vivo. As a proof of concept we show that catalytically inactive AGO1 variants, known to be impaired in slicing (Baumberger and Baulcombe, 2005; Carbonell et al., 2012), are unable to drive the repression of the cleavable sensors (Figures $5 \mathrm{~A}, 5 \mathrm{~B}$ ) suggesting that repression in this case is mainly exerted by transcript cleavage. This is further supported by our qRT-PCR analyses, which reveal that most of the repression observed at the level of LUC activity can be explained by cleavage of the LUC transcript (Figures 4A-4D). This is consistent with recent in vitro studies showing that the position and number of copies of the miRNA target site largely determine the mode of repression (Iwakawa and Tomari, 2013). Iwakawa and Tomari show that single miRNA target sites in the $3^{\prime} \mathrm{UTR}$, as employed here, result mostly in transcript cleavage, whereas multiple consecutive sites in the 3'UTR, as well as single sites in the 5'UTR or the ORF have very strong translational effects. Therefore by combining our sensors to modified variants bearing the miRNA target site on the 5'UTR one could have an in vivo system that easily discriminates between transcript cleavage and translational repression. Our conclusion is also in line with a recent study on the miR159MYB33/MYB65 regulatory module (Li et al., 2014), which shows that transcript cleavage prevails over translation repression when there is perfect central complementarity, even though this is not an essential condition for slicing. 
Our system could also be employed for assessing the impact of specific protein domains or post-translational modifications on miRNA activity. Phosphorylation is emerging as an important regulatory mechanism of the miRNA pathway (de la Fuente van Bentem et al., 2008; Horman et al., 2013; Jones et al., 2009; Manavella et al., 2012; Rudel et al., 2011; Shen et al., 2013; Wang et al., 2013; Zeng et al., 2008) and sensitive and dynamic methods are required to evaluate the impact of these modifications on miRNA activity.

Importantly, protoplasts can be prepared from different mutants and used to test the involvement of the respective components in the miRNA pathway. As an example, by isolating protoplasts from $d c / 2-1, d c / 4-2$, and $r d r 6-15$ mutants, we excluded the possibility that secondary siRNAs play a role in the repression of the reporter induced by AGO1 and miRNA (Figure S9). We further monitored miRNA activity in protoplasts from dc/1-9. As expected, not only endogenous miRNA activity was lower in dc/1-9, but also the combined overexpression of primary miRNA with AGO1 in this background was inefficient in repressing our miRNA sensors (Figure 6), unless the mutant was transiently complemented with DCL1.

In most cases, miRNA reporters have previously been used in plants to generate stable transgenic lines, which were subsequently used for imaging (Marin et al., 2010; Nodine and Bartel, 2010; Parizotto et al., 2004; Schwab et al., 2009). Even though these approaches have the advantage of being sensitive to tissue-specific miRNA activity, they are more time-consuming and often not quantitative. More recently, GUS-based miRNA sensors were used for a quantitative readout of miR159 activity (Li et al., 2014), but again this relied on the more tedious generation of stable transgenic lines. In another study, accumulation of TAS1 transcript and tasiR255 derived from the expression of a construct sensitive to an artificial miRNA (amiR173) in N. benthamiana leaves was used as proxy of amiR173 activity (Carbonell et al., 2012). Using accumulation of TAS transcripts and tasiRNAs to quantify miRNA activity is rather laborious and has pitfalls, such as the requirement of multiple processing steps for the formation of the final readout, tasiRNAs. Our approach relies on simple cloning steps and on a fast transient assay using Arabidopsis protoplasts 
to measure luciferase activity as a quantitative readout of miRNA activity. Very recently, an independent miRNA system employing luciferase-based reporters in $N$. benthamiana was developed with the purpose of unraveling complementarity requirements between miRNAs and their targets (Liu et al., 2014). The authors show that differences in complementarity within the target site impact on the efficacy of silencing, even when the target site is isolated from its "natural" sequence context (Liu et al., 2014). Our results are not only consistent with the observations made in this study, but also combine the advantages of an in vivo transient system with all the genetic tools available for Arabidopsis.

Recently, at least two sensor library systems to quantify miRNA activity in a high throughput manner have been developed in mammals (Mullokandov et al., 2012; Tian et al., 2012). These systems can be used for monitoring miRNA activities in response to various stimuli, such as chemical compounds or environmental conditions. In this context, we show that HSP90 inhibitors block miRNA activity (Figures 5C, 5D), most likely because HSP90 is required for the proper assembly and function of the RISC complex (Iki et al., 2010; Iwasaki et al., 2010; Miyoshi et al., 2010). We envision that our miRNA sensor system could hence serve as basis to generate libraries for high throughput miRNA activity analyses in plants. Furthermore, different treatments such as hormones, metabolites or abiotic stimuli may be applied to protoplasts transfected with sensors and, in this way, explore the regulation of miRNAs as a first step to assess their involvement in specific signaling cascades and cellular processes.

\section{Methods}

All primers used in this work are listed in Table S1.

\section{Plant growth}

All Arabidopsis (Arabidopsis thaliana) plants were in Columbia-O (Col-O) background. Sterilized seeds of WT, dc/2-1 (Xie et al., 2004), dc/4-2 (Xie et al., 2005), rdr6-15 (Allen et al., 2004) were stratified in the dark at $4^{\circ} \mathrm{C}$ for 2 days and sowed in pots containing a 1:3 vermiculite:soil mixture. For protoplast 
isolation of dc/1-9, sterilized and stratified seeds of dc/1-9 (Vazquez et al., 2004), and WT as control were sowed on plates containing 0.5X Murashige and Skoog medium with $0.1 \%$ MES, $0.8 \%$ phytoagar, and $1 \%$ sucrose. After approximately 10 days of growth, homozygous dc/1-9 were readily distinguishable by their phenotype and were transferred, in parallel with WT seedlings, to pots with a 1:3 vermiculite:soil mixture. Plants were grown throughout development under a photoperiod of $12 \mathrm{~h}$ light $\left(100 \mu \mathrm{E} ; 22^{\circ} \mathrm{C}\right) / 12 \mathrm{~h}$ dark $\left(18^{\circ} \mathrm{C}\right)$. Leaves were harvested for protoplast isolation $2 \mathrm{~h}$ after the onset of the light period.

\section{Cloning and preparation of constructs for protoplast transfection}

All constructs are listed in Table S2.

Sensor constructs were made in a pUC18-derived vector expressing firefly luciferase under the $35 \mathrm{~S}$ promoter and NOS terminator (Luehrsen et al., 1992), and inserting selected cleavable or non-cleavable miRNA targets sites in the 3'UTR by site-directed mutagenesis. AGO1 (AT1G48410) and DCL1 (AT1G01040) were expressed under the 35S promoter in p35S-HA-GW (Ehlert et al., 2006; Weltmeier et al., 2006). AGO1 mutations were generated on the previous construct by site-directed mutagenesis. pri319a (AT4G23713) was amplified from genomic DNA as described (Schwab et al., 2006). pri159a (AT1G73687) and pri161 (AT1G48267) were amplified as genomic DNA sequences encompassing 250 bp both 5' and 3' of the mature miRNA (Cuperus et al., 2010). All amplified primary miRNAs were introduced in the pHBT95 vector (Yoo et al., 2007) and expressed under the 35S promoter. Previously described pGREEN-IPS1 (AT3G09922) constructs bearing the target mimics for miR161.1 and miR161.2 (Todesco et al., 2010) were used as templates to amplify MIM161.1 and MIM161.2 with primers IPS1_BamHI_A and IPS1_Pstl_B (Table S1), and were thereafter inserted into the pHBT95 vector. IPS1 harboring MIM161.2 in pHBT95 was mutated using primers described elsewhere [(Todesco et al., 2010); Table S1] to generate MIM159 and MIM319.

Plasmid DNA for protoplast transfection was purified from $\mathrm{CsCl}$-gradients and stained with a RedSafe ${ }^{\mathrm{TM}}$ Nucleic Acid Staining Solution (ChemBio; 1:500). 


\section{Isolation and transfection of Arabidopsis protoplasts}

Protoplasts were isolated from mature fully-expanded leaves from 5-week-old plants as described (Baena-Gonzalez et al., 2007; Yoo et al., 2007). For small RNA blots, qRT-PCR experiments, and miRNA activity assays $12 \times 10^{5}, 2-4 \times 10^{5}$ or $2 \times 10^{4}$ protoplasts were transfected, respectively, using a ratio of $1 \mu \mathrm{g}$ plasmid DNA per $1 \times 10^{3}$ transfected protoplasts. In the miRNA activity assays, 8-10 $\mu \mathrm{g}$ of sensor construct were used, in combination with 12-10 $\mathrm{mg}$ of effector(s) constructs and $1 \mu \mathrm{g}$ of 35S::GUS (Baena-Gonzalez et al., 2007) as transfection control. In the assays for the relative quantification of LUC expression levels, the same components were scaled-up at least 10x, in order to maintain the ratio of plasmid DNA per transfected protoplasts. In both cases, a mER7 plasmid was used as control DNA (Kovtun et al., 1998). After transfection, protoplasts were incubated overnight under light $\left(15 \mu \mathrm{E} ; 25^{\circ} \mathrm{C}\right)$. To test the impact of chemicals on miRNA activity, the HSP90 inhibitors geldanamycin and radicicol (Iki et al., 2010; Iwasaki et al., 2010; Miyoshi et al., 2010), as well as the cysteine protease inhibitor E64d (Asanuma et al., 2003), were used at $2 \mu \mathrm{M}$ throughout the whole incubation; 0.5\% DMSO was used as mock. On the following day, protoplasts were harvested by centrifugation at $100 \mathrm{~g}$ for $3 \mathrm{~min}$, flash-frozen in dry ice, used immediately for RNA extraction or kept at $-20^{\circ} \mathrm{C}$ for luciferase and $\beta$-glucuronidase analyses (Yoo et al., 2007). To compare miRNA activity amongst different samples, luciferase activity values were normalized to $\beta$-glucuronidase activity derived from the co-transfected 35S::GUS reporter.

\section{RNA extraction}

RNA was extracted with TRizol (Life Sciences), according to the manufacturer's instructions. For $2-12 \times 10^{5}$ protoplasts, $500 \mu \mathrm{L}$ TRizol were used per extraction. Prior to cDNA synthesis, RNA was treated with RQ1 RNase-Free DNase (Promega; 0.5-3 DNase units per $\mu \mathrm{g}$ of RNA) at $37^{\circ} \mathrm{C}$ for $30 \mathrm{~min}$. In those cases where we quantified LUC expression levels by qPCR, RNA was treated twice with RQ1 RNase-Free DNase (Promega). After the DNase treatment, the RNA was recovered by phenol:chloroform precipitation. 


\section{Small RNA Blots}

We used small RNA blots to detect miRNAs following a published protocol (Varallyay et al., 2008), but substituting LNA probes by regular DNA oligonucleotides. The sequences of all probes are listed in Table S1. Owing to the high sequence similarity between miR159 and miR319, the miR159 probe was used for the simultaneous detection of both miRNAs, with miR319 being detected as a faster migrating band.

\section{Gene expression analyses}

DNase-treated RNA $(0.1-1 \mu \mathrm{g})$ was reverse transcribed using SuperScript III Reverse Transcriptase (Life Technologies), following the manufacturers' instructions. qRT-PCR analyses were performed either in a 7900HT Fast RealTime PCR System (Applied Biosystems) or a CFX384 ${ }^{\mathrm{TM}}$ Real-Time System (Bio-Rad), using the $\mathrm{iTaq}^{\mathrm{TM}}$ Universal $\mathrm{SYBR}{ }^{\mathrm{B}}$ Green Supermix (BioRad), and the $2^{-\Delta \Delta C t}$ method for relative quantification (Livak and Schmittgen, 2001). Unless indicated otherwise, expression values were normalized using the CT values obtained for the ACT2 (AT3G18780) reference gene. LUC expression values were normalized using co-transfected $\beta-G U S$ as a reference gene.

\section{Protein expression analyses}

Frozen pellets of transfected protoplasts were directly resuspended in $4 \mathrm{x}$ Laemmli solubilization buffer and loaded into an $8 \%$ SDS-PAGE gel. Proteins were transferred to a PVDF membrane using a Mini Trans-Blot $₫$ Cell (Bio-Rad), following the manufacturer's instructions. HA-tagged AGO1 proteins were detected using a High-Affinity anti-HA antibody (Roche).

\section{Supplementary Data}

Figure S1. Specific probes distinguish between synthetic oligonucleotides 
corresponding to the sequences of miR161.1 and miR161.2.

Figure S2. Relative quantification of TCP2 and TCP4 in stable plant lines overexpressing a miR319 target mimic (MIM319).

Figure S3. Overexpression of primary miRNAs 159 and 319 does not affect $P P D K$ and $A L D H 22 A 1$ levels.

Figure S4. Overexpression of target mimics for miR159 (MIM159) and miR319 (MIM319) does not affect PPDK and ALDH22A1 levels.

Figure S5. Firefly luciferase (fLUC) miRNA sensor to monitor miRNA 161.2 activity in Arabidopsis protoplasts.

Figure S6. Non-cleavable miRNA sensor for miR161.2 behaves essentially as a variant with higher number of mutations.

Figure S7. Luciferase-based miRNA sensors are highly specific tools to measure miRNA activity in Arabidopsis protoplasts.

Figure S8. Basal activities of cleavable and non-cleavable reporters in WT and dc/1-9.

Figure S9. Repression of miRNA reporters does not require the formation of secondary siRNAs.

Table S1. List of primers used.

Table S2. List of constructs used.

\section{Acknowledgements}

Vera Nunes is thanked for excellent plant care. Seeds for the various mutants were kindly provided by Hervé Vaucheret (dc/1-9), Steven Jacobsen ( $d c / 2-1)$, and James Carrington (dc/4-2 and rdr6-15). Guillaume Tena (Massachusetts General Hospital, Boston, USA) is thanked for the 35S::pri319a construct. CM, $A C$ and $P C$ were supported by Fundação para a Ciência e Tecnologia fellowships SFRH/BD/33563/2008, SFRH/BPD/47280/2008 and SFRH/BPD/79255/2011, respectively. IRS was supported by a fellowship from EMBO. DW was supported by grants from the Deutsche Forschungsgemeinschaft (SPP1530) and the Max Planck Society. EBG was supported by grants from the EMBO Installation program, and Fundação para 
a Ciência e a Tecnologia (FCT- PTCD/BIA-BCM/107924/2008). The authors declare no conflict of interest.

\section{Figure legends}

Figure 1. Primary miRNAs are processed into functional mature miRNAs in Arabidopsis protoplasts. A, Transient overexpression of primary miRNAs results in MIR transcript processing and mature miRNA overexpression, as detected by small RNA blots. Note that both miR159 and miR319 are recognized by a probe against miR159 due to the high sequence similarity of the two miRNAs. The low levels of endogenous miR319 preclude its detection. U6, loading control. B, Overexpression of pri319, but not of sequence-related pri159 nor sequence-unrelated pri161, triggers repression of TCP2 and TCP4, as quantified by qRT-PCR. C, Overexpression of pri159, pri319 and pri161 does not significantly alter MYB33 and MYB65, as quantified by qRT-PCR. ACT2, reference gene. Graphs represent mean \pm SEM of at least three independent biological replicates. Numbers refer to $p$ values, obtained by a ratio paired $t$-test comparing each primary miRNA vs. a control plasmid. ns, non significant.

Figure 2. Overexpression of miRNA target mimics efficiently suppresses miRNA function in Arabidopsis protoplasts. A, Transient overexpression of miRNA target mimics (MIMs) decreases the levels of the corresponding mature miRNAs, as detected by small RNA blots. U6, loading control. B, Overexpression of MIM319 induces TCP4 accumulation. TCP4 accumulates to a lower extent also in the presence of the sequence-related MIM159, but not in the presence of the sequence-unrelated MIM161.1 and MIM161.2. TCP2 expression is not significantly affected by any of the target mimics. C, Overexpression of MIM159 induces a 10-fold increase in MYB33 and MYB65 levels. Overexpression of MIM319 also induces MYB33 and MYB65, but to a minor extent, whilst MIM161.1 and MIM161.2 have negligible effects. Relative quantification by qRT-PCR. ACT2, reference gene. Graphs represent mean \pm SEM of at least three independent biological replicates. Numbers refer to 
$p$ values, obtained by ratio paired $t$-test comparing each target mimic $v s$. a control plasmid. $n s$, non significant.

Figure 3. Firefly luciferase ( $f L U C$ ) miRNA sensors to monitor miRNA activity in Arabidopsis protoplasts. The TCP4 and MYB33 target sites for miR319 and for miR159, respectively, were introduced in the 3'UTR of $f L U C$, generating the corresponding cleavable reporters. As negative controls, noncleavable sensor variants were produced harboring mutations in the positions corresponding to nucleotides 10 and 11 of the respective miRNAs (shown in red). In all cases, expression of the $f L U C$ coding sequence (CDS) is under the control of the $35 S$ promoter and the NOS terminator.

Figure 4. Luciferase-based miRNA sensors are highly specific and sensitive tools to measure miRNA activity in Arabidopsis protoplasts.

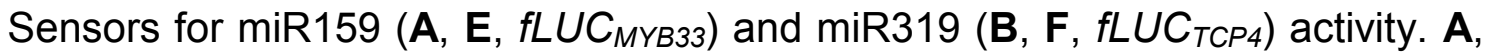
B, Graphs depict normalized luciferase activity of cleavable $(C-f L U C)$ vs. noncleavable (NC-fLUC) variants in the presence of the indicated components; $p$ values, two-way ANOVA and Fisher's LSD test. C, D, Co-expression of AGO1 and miRNA causes a decrease in the transcripts of the cleavable fLUC reporters, whilst having no impact on the non-cleavable reporter variants. LUC transcript levels were measured by qRT-PCR using primers flanking the miRNA target site and normalized to the levels of a co-expressed 35S::GUS transcript. $p$ values, two-way ANOVA and Fisher's LSD test. E, F, Graphs depict normalized luciferase activity of cleavable variants $(C-f L U C)$ in the presence of the indicated components. Significantly different pairs of bars are marked by the same letter, according to $p$ values obtained by one-way ANOVA and Fisher's LSD test. Bars represent mean \pm SEM of at least three independent experiments. ns, non significant.

Figure 5. Use of miRNA sensors for dissection of miRNA pathways: functional studies on the silencing machinery. A, B, Catalytically inactive AGO1 variants are unable to repress the miRNA sensors. Graphs depict 
normalized luciferase activity of cleavable variants $(C-f L U C)$ of sensors for miR159 (A, C- $\left.f L U C_{M Y B 33}\right)$ and miR319 (B, C-fLUC $\left.C_{T C P 4}\right)$ activity in the presence of the indicated miRNA and WT or mutated AGO1 protein. All AGO1 variants bear an HA-tag and their expression was confirmed by immunoblotting with an HA-antibody. Membranes were stained with Coomassie Blue for loading control. $p$ values, ratio paired $t$-test of the indicated sample pairs. C, D, HSP90 inhibitors (geldanamycin and radicicol) inhibit miRNA activity whilst a cysteine protease inhibitor (E64d) has no effect. Graphs depict normalized luciferase activity of cleavable variants $(C-f L U C)$ of miR159 (C, C-fLUC $C_{M Y B 33)}$ and miR319 (D, C$\left.f L U C_{T C P 4}\right)$ sensors. Bars represent mean $\pm S E M$ of three independent experiments. $n s$, non significant.

Figure 6. Use of miRNA sensors for dissection of miRNA pathways: monitoring miRNA activity in the dcl1-9 mutant. Graphs depict normalized luciferase activity of cleavable variants $(C$-fLUC) of sensors for miR159 (A, C$\left.f L U C_{M Y B 33}\right)$ and miR319 (B, C-fLUC $\left.C_{T C P 4}\right)$ activity in the dc/1-9 mutant and WT plants. $p$ values, two-way ANOVA and Fisher's LSD test. Bars represent mean $\pm S E M$ of three independent experiments. $n s$, non significant.

\section{References}

Achard P, Herr A, Baulcombe DC, Harberd NP. Modulation of floral development by a gibberellin-regulated microRNA. Development (2004) 131:3357-3365.

Allen E, Xie Z, Gustafson AM, Carrington JC. microRNA-directed phasing during transacting siRNA biogenesis in plants. Cell (2005) 121:207-221.

Allen E, Xie Z, Gustafson AM, Sung GH, Spatafora JW, Carrington JC. Evolution of microRNA genes by inverted duplication of target gene sequences in Arabidopsis thaliana. Nat Genet (2004) 36:1282-1290.

Alonso-Peral MM, Sun C, Millar AA. MicroRNA159 can act as a switch or tuning microRNA independently of its abundance in Arabidopsis. PLoS One (2012) 7:e34751.

Ameres SL, Zamore PD. Diversifying microRNA sequence and function. Nat Rev Mol Cell Biol (2013) 14:475-488.

Asanuma $\mathrm{K}$, et al. MAP-LC3, a promising autophagosomal marker, is processed during the differentiation and recovery of podocytes from PAN nephrosis. FASEB J (2003) 17:1165-1167.

Aukerman MJ, Sakai $\mathrm{H}$. Regulation of flowering time and floral organ identity by a MicroRNA and its APETALA2-like target genes. Plant Cell (2003) 15:27302741. 
Axtell MJ. Classification and comparison of small RNAs from plants. Annu Rev Plant Biol (2013) 64:137-159.

Baek D, Villen J, Shin C, Camargo FD, Gygi SP, Bartel DP. The impact of microRNAs on protein output. Nature (2008) 455:64-71.

Baena-Gonzalez E, Rolland F, Thevelein JM, Sheen J. A central integrator of transcription networks in plant stress and energy signalling. Nature (2007) 448:938-942.

Baumberger N, Baulcombe DC. Arabidopsis ARGONAUTE1 is an RNA Slicer that selectively recruits microRNAs and short interfering RNAs. Proc Natl Acad Sci U S A (2005) 102:11928-11933.

Beillard E, Ong SC, Giannakakis A, Guccione E, Vardy LA, Voorhoeve PM. miR-Sens-a retroviral dual-luciferase reporter to detect microRNA activity in primary cells. RNA (2012) 18:1091-1100.

Brodersen $\mathrm{P}$, et al. Widespread translational inhibition by plant miRNAs and siRNAs. Science (2008) 320:1185-1190.

Brodersen $\mathrm{P}$, et al. Isoprenoid biosynthesis is required for miRNA function and affects membrane association of ARGONAUTE 1 in Arabidopsis. Proc Natl Acad Sci U S A (2012) 109:1778-1783.

Brodersen $\mathrm{P}$, Voinnet $\mathrm{O}$. Revisiting the principles of microRNA target recognition and mode of action. Nat Rev Mol Cell Biol (2009) 10:141-148.

Brown BD, et al. Endogenous microRNA can be broadly exploited to regulate transgene expression according to tissue, lineage and differentiation state. Nat Biotechnol (2007) 25:1457-1467.

Burchard $\mathrm{J}$, et al. MicroRNA-like off-target transcript regulation by siRNAs is species specific. RNA (2009) 15:308-315.

Carbonell A, et al. Functional analysis of three Arabidopsis ARGONAUTES using slicer-defective mutants. Plant Cell (2012) 24:3613-3629.

Carlsbecker A, et al. Cell signalling by microRNA165/6 directs gene dose-dependent root cell fate. Nature (2010) 465:316-321.

Cho $\mathrm{HJ}$, et al. SHORT VEGETATIVE PHASE (SVP) protein negatively regulates miR172 transcription via direct binding to the pri-miR172a promoter in Arabidopsis. FEBS Lett (2012) 586:2332-2337.

Confraria A, Martinho C, Elias A, Rubio-Somoza I, Baena-Gonzalez E. miRNAs mediate SnRK1-dependent energy signaling in Arabidopsis. Front Plant Sci (2013) 4:197.

Connelly CM, Thomas M, Deiters A. High-throughput luciferase reporter assay for small-molecule inhibitors of microRNA function. J Biomol Screen (2012) 17:822828.

Cuperus JT, et al. Identification of MIR390a precursor processing-defective mutants in Arabidopsis by direct genome sequencing. Proc Natl Acad Sci U S A (2010) 107:466-471.

Dai X, Zhao PX. psRNATarget: a plant small RNA target analysis server. Nucleic Acids Res (2011) 39:W155-159.

de la Fuente van Bentem S, et al. Site-specific phosphorylation profiling of Arabidopsis proteins by mass spectrometry and peptide chip analysis. J Proteome Res (2008) 7:2458-2470.

Derrien $B$, et al. Degradation of the antiviral component ARGONAUTE1 by the autophagy pathway. Proc Natl Acad Sci U S A (2012) 109:15942-15946.

Doench JG, Sharp PA. Specificity of microRNA target selection in translational repression. Genes Dev (2004) 18:504-511.

Ehlert A, et al. Two-hybrid protein-protein interaction analysis in Arabidopsis protoplasts: establishment of a heterodimerization map of group $\mathrm{C}$ and group $\mathrm{S}$ bZIP transcription factors. Plant J (2006) 46:890-900. 
Fahlgren N, et al. High-throughput sequencing of Arabidopsis microRNAs: evidence for frequent birth and death of MIRNA genes. PLoS One (2007) 2:e219.

Folkes L, et al. PAREsnip: a tool for rapid genome-wide discovery of small RNA/target interactions evidenced through degradome sequencing. Nucleic Acids Res (2012) 40:e103.

Franco-Zorrilla JM, et al. Target mimicry provides a new mechanism for regulation of microRNA activity. Nat Genet (2007) 39:1033-1037.

Gibbings D, Mostowy S, Jay F, Schwab Y, Cossart P, Voinnet O. Selective autophagy degrades DICER and AGO2 and regulates miRNA activity. Nat Cell Biol (2012) 14:1314-1321.

Grimson A, Farh KK, Johnston WK, Garrett-Engele P, Lim LP, Bartel DP. MicroRNA targeting specificity in mammals: determinants beyond seed pairing. Mol Cell (2007) 27:91-105.

Gu S, Jin L, Zhang F, Sarnow P, Kay MA. Biological basis for restriction of microRNA targets to the $3^{\prime}$ untranslated region in mammalian mRNAs. Nat Struct Mol Biol (2009) 16:144-150.

Hajdarpasic A, Ruggenthaler P. Analysis of miRNA expression under stress in Arabidopsis thaliana. Bosn J Basic Med Sci (2012) 12:169-176.

Horman SR, et al. Akt-mediated phosphorylation of argonaute 2 downregulates cleavage and upregulates translational repression of MicroRNA targets. Mol Cell (2013) 50:356-367.

Hsieh LC, et al. Uncovering small RNA-mediated responses to phosphate deficiency in Arabidopsis by deep sequencing. Plant Physiol (2009) 151:2120-2132.

Huang PC, Chen CY, Yang FY, Au LC. A multisampling reporter system for monitoring microRNA activity in the same population of cells. J Biomed Biotechnol (2009) 2009:104716.

Humphreys DT, Westman BJ, Martin DI, Preiss T. MicroRNAs control translation initiation by inhibiting eukaryotic initiation factor 4E/cap and poly $(A)$ tail function. Proc Natl Acad Sci U S A (2005) 102:16961-16966.

Iki T, Yoshikawa M, Meshi T, Ishikawa M. Cyclophilin 40 facilitates HSP90-mediated RISC assembly in plants. EMBO J (2012) 31:267-278.

Iki $\mathrm{T}$, et al. In vitro assembly of plant RNA-induced silencing complexes facilitated by molecular chaperone HSP90. Mol Cell (2010) 39:282-291.

Iwakawa HO, Tomari Y. Molecular Insights into microRNA-Mediated Translational Repression in Plants. Mol Cell (2013) 52:591-601.

Iwasaki S, et al. Hsc70/Hsp90 chaperone machinery mediates ATP-dependent RISC loading of small RNA duplexes. Mol Cell (2010) 39:292-299.

Janas MM, et al. Reduced expression of ribosomal proteins relieves microRNAmediated repression. Mol Cell (2012) 46:171-186.

Jeong $\mathrm{DH}$, et al. Massive analysis of rice small RNAs: mechanistic implications of regulated microRNAs and variants for differential target RNA cleavage. Plant Cell (2011) 23:4185-4207.

Jones-Rhoades MW, Bartel DP. Computational identification of plant microRNAs and their targets, including a stress-induced miRNA. Mol Cell (2004) 14:787-799.

Jones-Rhoades MW, Bartel DP, Bartel B. MicroRNAS and their regulatory roles in plants. Annu Rev Plant Biol (2006) 57:19-53.

Jones AM, et al. Phosphoproteomic analysis of nuclei-enriched fractions from Arabidopsis thaliana. J Proteomics (2009) 72:439-451.

Jung JH, Seo PJ, Ahn JH, Park CM. Arabidopsis RNA-binding protein FCA regulates microRNA172 processing in thermosensory flowering. J Biol Chem (2012) 287:16007-16016. 
Khan AA, Betel D, Miller ML, Sander C, Leslie CS, Marks DS. Transfection of small RNAs globally perturbs gene regulation by endogenous microRNAs. Nat Biotechnol (2009) 27:549-555.

Kovtun Y, Chiu WL, Zeng W, Sheen J. Suppression of auxin signal transduction by a MAPK cascade in higher plants. Nature (1998) 395:716-720.

Kozomara A, Griffiths-Jones S. miRBase: integrating microRNA annotation and deepsequencing data. Nucleic Acids Res (2011) 39:D152-157.

Krutzfeldt J, et al. Silencing of microRNAs in vivo with 'antagomirs'. Nature (2005) 438:685-689.

Kurihara Y, Watanabe Y. Arabidopsis micro-RNA biogenesis through Dicer-like 1 protein functions. Proc Natl Acad Sci U S A (2004) 101:12753-12758.

Lanet $\mathrm{E}$, et al. Biochemical evidence for translational repression by Arabidopsis microRNAs. Plant Cell (2009) 21:1762-1768.

Laubinger $S$, et al. Global effects of the small RNA biogenesis machinery on the Arabidopsis thaliana transcriptome. Proc Natl Acad Sci U S A (2010) 107:17466-17473.

Leung AK, Sharp PA. microRNAs: a safeguard against turmoil? Cell (2007) 130:581585.

Li J, Reichel M, Millar AA. Determinants beyond both complementarity and cleavage govern microR159 efficacy in Arabidopsis. PLoS Genet (2014) 10:e1004232.

Li JF, Chung HS, Niu Y, Bush J, McCormack M, Sheen J. Comprehensive proteinbased artificial microRNA screens for effective gene silencing in plants. Plant Cell (2013a) 25:1507-1522.

$\mathrm{Li} S$, et al. MicroRNAs inhibit the translation of target mRNAs on the endoplasmic reticulum in Arabidopsis. Cell (2013b) 153:562-574.

Liang $\mathrm{G}, \mathrm{He} \mathrm{H}, \mathrm{Yu} \mathrm{D}$. Identification of nitrogen starvation-responsive microRNAs in Arabidopsis thaliana. PLoS One (2012) 7:e48951.

Lim LP, et al. Microarray analysis shows that some microRNAs downregulate large numbers of target mRNAs. Nature (2005) 433:769-773.

Liu HH, Tian X, Li YJ, Wu CA, Zheng CC. Microarray-based analysis of stressregulated microRNAs in Arabidopsis thaliana. RNA (2008) 14:836-843.

Liu Q, Wang F, Axtell MJ. Analysis of complementarity requirements for plant MicroRNA targeting using a Nicotiana benthamiana quantitative transient assay. Plant Cell (2014) 26:741-753.

Liu Q, Yao X, Pi L, Wang H, Cui X, Huang H. The ARGONAUTE10 gene modulates shoot apical meristem maintenance and establishment of leaf polarity by repressing miR165/166 in Arabidopsis. Plant J (2009) 58:27-40.

Livak KJ, Schmittgen TD. Analysis of relative gene expression data using real-time quantitative PCR and the $2^{-\Delta \Delta C T}$ method. Methods (2001) 25:402-408.

Llave C, Xie Z, Kasschau KD, Carrington JC. Cleavage of Scarecrow-like mRNA targets directed by a class of Arabidopsis miRNA. Science (2002) 297:20532056.

Luehrsen KR, de Wet JR, Walbot V. Transient expression analysis in plants using firefly luciferase reporter gene. Methods Enzymol (1992) 216:397-414.

Lv DK, et al. Profiling of cold-stress-responsive miRNAs in rice by microarrays. Gene (2010) 459:39-47.

Lytle JR, Yario TA, Steitz JA. Target mRNAs are repressed as efficiently by microRNAbinding sites in the 5' UTR as in the 3' UTR. Proc Natl Acad Sci U S A (2007) 104:9667-9672.

Mallory AC, Elmayan T, Vaucheret $\mathrm{H}$. MicroRNA maturation and action--the expanding roles of ARGONAUTEs. Curr Opin Plant Biol (2008) 11:560-566.

Manavella PA, et al. Fast-forward genetics identifies plant CPL phosphatases as regulators of miRNA processing factor HYL1. Cell (2012) 151:859-870. 
Marin E, et al. miR390, Arabidopsis TAS3 tasiRNAs, and their AUXIN RESPONSE FACTOR targets define an autoregulatory network quantitatively regulating lateral root growth. Plant Cell (2010) 22:1104-1117.

Mi S, et al. Sorting of small RNAs into Arabidopsis argonaute complexes is directed by the $5^{\prime}$ terminal nucleotide. Cell (2008) 133:116-127.

Millar AA, Gubler F. The Arabidopsis GAMYB-like genes, MYB33 and MYB65, are microRNA-regulated genes that redundantly facilitate anther development. Plant Cell (2005) 17:705-721.

Miyoshi T, Takeuchi A, Siomi H, Siomi MC. A direct role for Hsp90 in pre-RISC formation in Drosophila. Nat Struct Mol Biol (2010) 17:1024-1026.

Moldovan D, Spriggs A, Yang J, Pogson BJ, Dennis ES, Wilson IW. Hypoxiaresponsive microRNAs and trans-acting small interfering RNAs in Arabidopsis. J Exp Bot (2010) 61:165-177.

Moxon S, et al. Deep sequencing of tomato short RNAs identifies microRNAs targeting genes involved in fruit ripening. Genome Res (2008a) 18:1602-1609.

Moxon S, Schwach F, Dalmay T, Maclean D, Studholme DJ, Moulton V. A toolkit for analysing large-scale plant small RNA datasets. Bioinformatics (2008b) 24:2252-2253.

Mullokandov G, et al. High-throughput assessment of microRNA activity and function using microRNA sensor and decoy libraries. Nat Methods (2012) 9:840-846.

Navarro L, et al. A plant miRNA contributes to antibacterial resistance by repressing auxin signaling. Science (2006) 312:436-439.

Nicolas FE. Experimental validation of microRNA targets using a luciferase reporter system. Methods Mol Biol (2011) 732:139-152.

Nodine MD, Bartel DP. MicroRNAs prevent precocious gene expression and enable pattern formation during plant embryogenesis. Genes Dev (2010) 24:26782692.

Palatnik JF, et al. Control of leaf morphogenesis by microRNAs. Nature (2003) 425:257-263.

Palatnik JF, et al. Sequence and expression differences underlie functional specialization of Arabidopsis microRNAs miR159 and miR319. Dev Cell (2007) 13:115-125.

Pant BD, et al. Identification of nutrient-responsive Arabidopsis and rapeseed microRNAs by comprehensive real-time polymerase chain reaction profiling and small RNA sequencing. Plant Physiol (2009) 150:1541-1555.

Parizotto EA, Dunoyer $P$, Rahm N, Himber $C$, Voinnet $O$. In vivo investigation of the transcription, processing, endonucleolytic activity, and functional relevance of the spatial distribution of a plant miRNA. Genes Dev (2004) 18:2237-2242.

Park W, Li J, Song R, Messing J, Chen X. CARPEL FACTORY, a Dicer homolog, and HEN1, a novel protein, act in microRNA metabolism in Arabidopsis thaliana. Curr Biol (2002) 12:1484-1495.

Petersen CP, Bordeleau ME, Pelletier J, Sharp PA. Short RNAs repress translation after initiation in mammalian cells. Mol Cell (2006) 21:533-542.

Ramachandran V, Chen X. Degradation of microRNAs by a family of exoribonucleases in Arabidopsis. Science (2008) 321:1490-1492.

Rogers K, Chen X. Biogenesis, turnover, and mode of action of plant microRNAs. Plant Cell (2013) 25:2383-2399.

Ronemus M, Vaughn MW, Martienssen RA. MicroRNA-targeted and small interfering RNA-mediated mRNA degradation is regulated by argonaute, dicer, and RNAdependent RNA polymerase in Arabidopsis. Plant Cell (2006) 18:1559-1574.

Rudel S, et al. Phosphorylation of human Argonaute proteins affects small RNA binding. Nucleic Acids Res (2011) 39:2330-2343. 
Ruiz-Ferrer V, Voinnet O. Roles of plant small RNAs in biotic stress responses. Annu Rev Plant Biol (2009) 60:485-510.

Schwab R, et al. Endogenous TasiRNAs mediate non-cell autonomous effects on gene regulation in Arabidopsis thaliana. PLoS One (2009) 4:e5980.

Schwab R, Ossowski S, Riester M, Warthmann N, Weigel D. Highly specific gene silencing by artificial microRNAs in Arabidopsis. Plant Cell (2006) 18:11211133.

Schwab R, Palatnik JF, Riester M, Schommer C, Schmid M, Weigel D. Specific effects of microRNAs on the plant transcriptome. Dev Cell (2005) 8:517-527.

Selbach M, Schwanhausser B, Thierfelder N, Fang Z, Khanin R, Rajewsky N. Widespread changes in protein synthesis induced by microRNAs. Nature (2008) 455:58-63.

Shen J, et al. EGFR modulates microRNA maturation in response to hypoxia through phosphorylation of AGO2. Nature (2013) 497:383-387.

Sunkar R, Chinnusamy V, Zhu J, Zhu JK. Small RNAs as big players in plant abiotic stress responses and nutrient deprivation. Trends Plant Sci (2007) 12:301-309.

Tang G, Reinhart BJ, Bartel DP, Zamore PD. A biochemical framework for RNA silencing in plants. Genes Dev (2003) 17:49-63.

Tian W, et al. High-throughput functional microRNAs profiling by recombinant AAVbased microRNA sensor arrays. PLoS One (2012) 7:e29551.

Todesco M, Rubio-Somoza I, Paz-Ares J, Weigel D. A collection of target mimics for comprehensive analysis of microRNA function in Arabidopsis thaliana. PLoS Genet (2010) 6:e1001031.

Varallyay E, Burgyan J, Havelda Z. MicroRNA detection by northern blotting using locked nucleic acid probes. Nat Protoc (2008) 3:190-196.

Vaucheret $H$, Vazquez F, Crete P, Bartel DP. The action of ARGONAUTE1 in the miRNA pathway and its regulation by the miRNA pathway are crucial for plant development. Genes Dev (2004) 18:1187-1197.

Vazquez $F$, et al. Endogenous trans-acting siRNAs regulate the accumulation of Arabidopsis mRNAs. Mol Cell (2004) 16:69-79.

Wang JW, Czech B, Weigel D. miR156-regulated SPL transcription factors define an endogenous flowering pathway in Arabidopsis thaliana. Cell (2009) 138:738749.

Wang JW, Schwab R, Czech B, Mica E, Weigel D. Dual effects of miR156-targeted SPL genes and CYP78A5/KLUH on plastochron length and organ size in Arabidopsis thaliana. Plant Cell (2008) 20:1231-1243.

Wang P, et al. Quantitative phosphoproteomics identifies SnRK2 protein kinase substrates and reveals the effectors of abscisic acid action. Proc Natl Acad Sci U S A (2013) 110:11205-11210.

Wang $W$, et al. An importin beta protein negatively regulates MicroRNA activity in Arabidopsis. Plant Cell (2011) 23:3565-3576.

Weltmeier $\mathrm{F}$, et al. Combinatorial control of Arabidopsis proline dehydrogenase transcription by specific heterodimerisation of bZIP transcription factors. EMBO J (2006) 25:3133-3143.

Wu G, Park MY, Conway SR, Wang JW, Weigel D, Poethig RS. The sequential action of miR156 and miR172 regulates developmental timing in Arabidopsis. Cell (2009) 138:750-759.

Xie Z, Allen E, Wilken A, Carrington JC. DICER-LIKE 4 functions in trans-acting small interfering RNA biogenesis and vegetative phase change in Arabidopsis thaliana. Proc Natl Acad Sci U S A (2005) 102:12984-12989.

Xie Z, et al. Genetic and functional diversification of small RNA pathways in plants. PLoS Biol (2004) 2:E104. 
Yan K, et al. Stress-induced alternative splicing provides a mechanism for the regulation of microRNA processing in Arabidopsis thaliana. Mol Cell (2012) 48:521-531.

Yoo SD, Cho YH, Sheen J. Arabidopsis mesophyll protoplasts: a versatile cell system for transient gene expression analysis. Nat Protoc (2007) 2:1565-1572.

Zeng Y, Sankala H, Zhang X, Graves PR. Phosphorylation of Argonaute 2 at serine387 facilitates its localization to processing bodies. Biochem J (2008) 413:429436.

Zhang H, Li L. SQUAMOSA promoter binding protein-like7 regulated microRNA408 is required for vegetative development in Arabidopsis. Plant J (2013) 74:98-109.

Zhu H, et al. Arabidopsis Argonaute10 specifically sequesters miR166/165 to regulate shoot apical meristem development. Cell (2011) 145:242-256.

Zhu QH, Helliwell CA. Regulation of flowering time and floral patterning by miR172. J Exp Bot (2011) 62:487-495. 
A
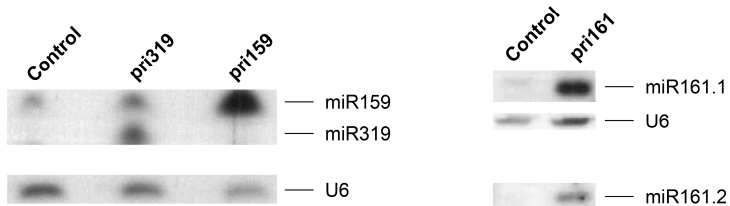

- - miR161.2

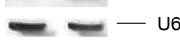

B

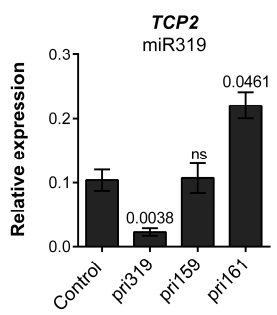

$T C P 4$

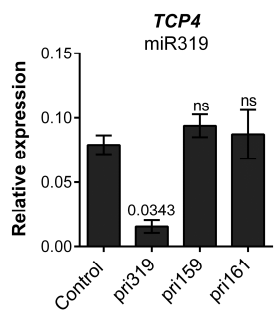

C
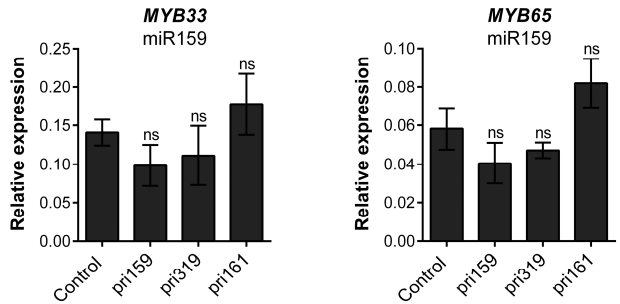

Figure 1. Primary miRNAs are processed into functional mature miRNAs in Arabidopsis protoplasts. A, Transient overexpression of primary miRNAs results in MIR transcript processing and mature miRNA overexpression, as detected by small RNA blots. Note that both miR159 and miR319 are recognized by a probe against miR159 due to the high sequence similarity of the two miRNAs. The low levels of endogenous miR319 preclude its detection. U6, loading control. B, Overexpression of pri319, but not of sequence-related pri159 nor sequenceunrelated pri161, triggers repression of TCP2 and TCP4, as quantified by qRT-PCR. C Overexpression of pri159, pri319 and pri161 does not significantly alter MYB33 and MYB65, as quantified by qRT-PCR. ACT2, reference gene. Graphs represent mean \pm SEM of at least three independent biological replicates. Numbers refer to $p$ values, obtained by a ratio paired $t$-test comparing each primary miRNA vs. a control plasmid. ns, non significant. 
Figure 2
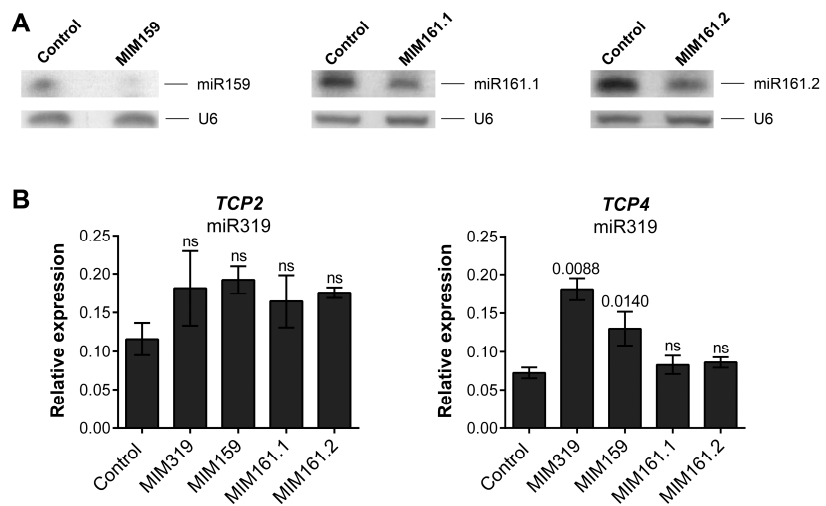

C
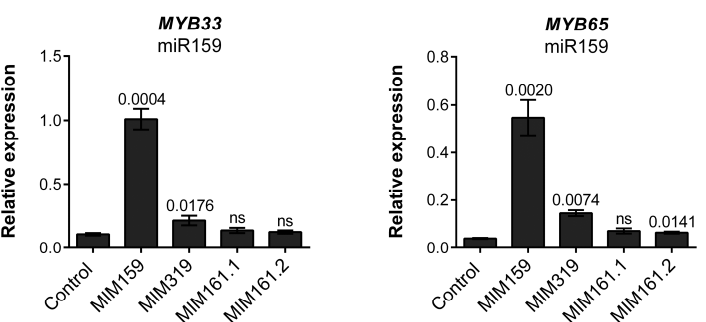

Figure 2. Overexpression of miRNA target mimics efficiently suppresses miRNA function in Arabidopsis protoplasts. A, Transient overexpression of miRNA target mimics (MIMs) decreases the levels of the corresponding mature miRNAs, as detected by small RNA blots. U6, loading control. B, Overexpression of MIM319 induces TCP4 accumulation. TCP4 accumulates to a lower extent also in the presence of the sequence-related MIM159, but not in the presence of the sequence-unrelated MIM161.1 and MIM161.2. TCP2 expression is not significantly affected by any of the target mimics. C, Overexpression of MIM159 induces a 10-fold increase in MYB33 and MYB65 levels. Overexpression of MIM319 also induces MYB33 and MYB65, but to a minor extent, whilst MIM161.1 and MIM161.2 have negligible effects. Relative quantification by qRT-PCR. ACT2, reference gene. Graphs represent mean $\pm S E M$ of at least three independent biological replicates. Numbers refer to $p$ values, obtained by ratio paired $t$-test comparing each target mimic vs. a control plasmid. ns, non significant. 


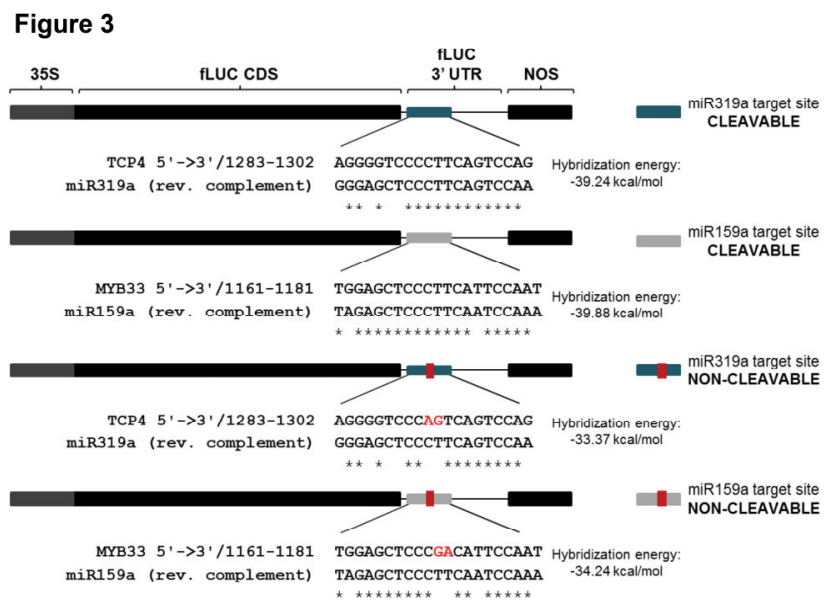

Figure 3. Firefly luciferase $(f L U C)$ miRNA sensors to monitor miRNA activity in Arabidopsis protoplasts. The TCP4 and MYB33 target sites for miR319 and for miR159, respectively, were introduced in the 3'UTR of $f L U C$, generating the corresponding cleavable reporters. As negative controls, non-cleavable sensor variants were produced harboring mutations in the positions corresponding to nucleotides 10 and 11 of the respective miRNAs (shown in red). In all cases, expression of the $f L U C$ coding sequence (CDS) is under the control of the $35 S$ promoter and the NOS terminator. 
Figure 4
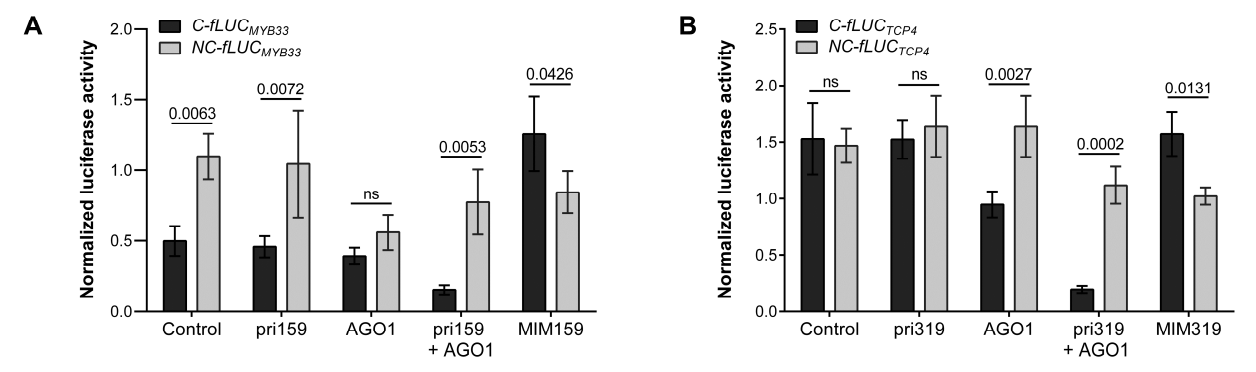

C

E
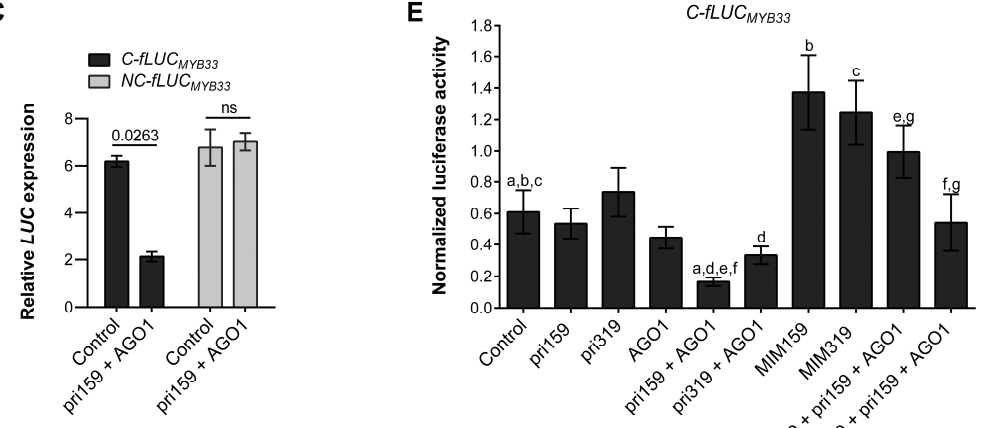

D

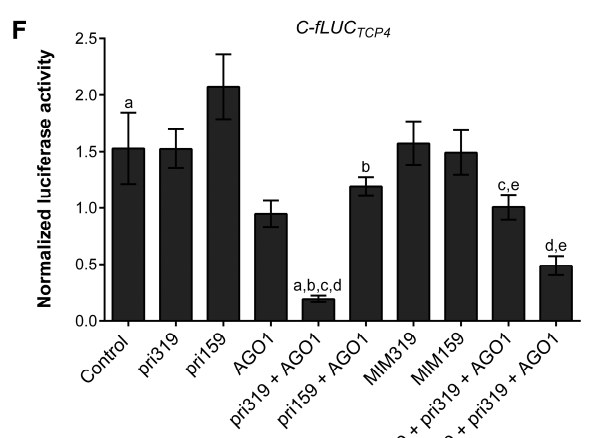

Figure 4. Luciferase-based miRNA sensors are highly specific and sensitive tools to measure miRNA activity in Arabidopsis protoplasts. Sensors for miR159 (A, E, fLUC ${ }_{M Y B 33}$ ) and miR319 (B, F, fLUC $C_{T C P 4}$ ) activity. A, B, Graphs depict normalized luciferase activity of cleavable $(C-f L U C)$ vs. non-cleavable $(N C$ - $f L U C)$ variants in the presence of the indicated components; $p$ values, two-way ANOVA and Fisher's LSD test. C, D, Co-expression of AGO1 and miRNA causes a decrease in the transcripts of the cleavable fLUC reporters, whilst having no impact on the non-cleavable reporter variants. LUC transcript levels were measured by qRTPCR using primers flanking the miRNA target site and normalized to the levels of a coexpressed 35S::GUS transcript. $p$ values, two-way ANOVA and Fisher's LSD test. E, F, Graphs depict normalized luciferase activity of cleavable variants $(C-f L U C)$ in the presence of the indicated components. Significantly different pairs of bars are marked by the same letter, according to $p$ values obtained by one-way ANOVA and Fisher's LSD test. Bars represent mean $\pm S E M$ of at least three independent experiments. $n s$, non significant. 
Figure 5
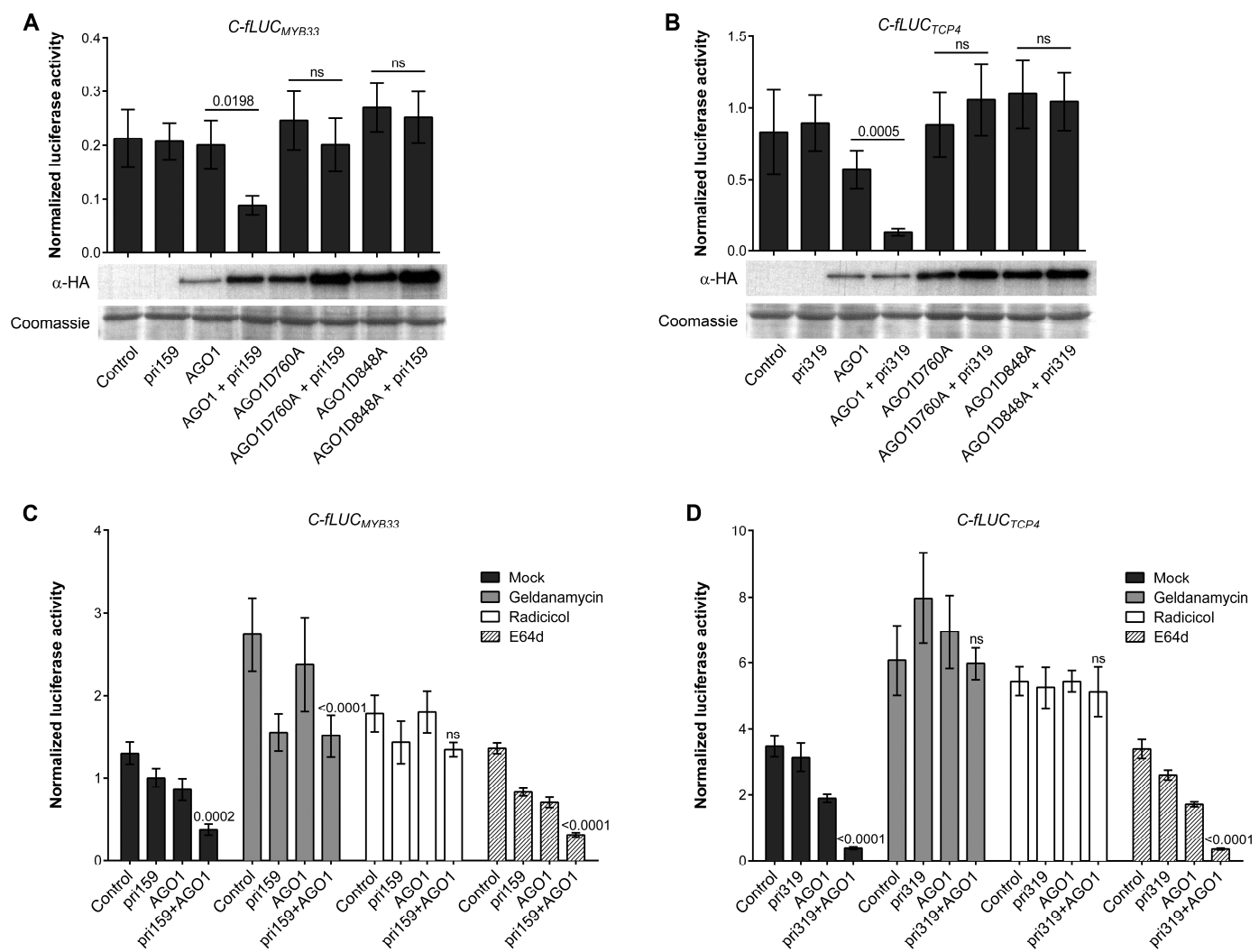

Figure 5. Use of miRNA sensors for dissection of miRNA pathways: functional studies on the silencing machinery. A, B, Catalytically inactive AGO1 variants are unable to repress the miRNA sensors. Graphs depict normalized luciferase activity of cleavable variants $(C$ - $f L U C)$ of sensors for miR159 (A, C-fLUC $C_{M Y B 33}$ ) and miR319 (B, C- $f L U C_{T C P 4}$ ) activity in the presence of the indicated miRNA and WT or mutated AGO1 protein. All AGO1 variants bear an HA-tag and their expression was confirmed by immunoblotting with an HA-antibody. Membranes were stained with Coomassie Blue for loading control. $p$ values, ratio paired $t$-test of the indicated sample pairs. C, D, HSP90 inhibitors (geldanamycin and radicicol) inhibit miRNA activity whilst a cysteine protease inhibitor (E64d) has no effect. Graphs depict normalized luciferase activity of cleavable variants (C-fLUC) of miR159 (C, C-fLUC $\left.C_{M Y B 33}\right)$ and miR319 (D, C- $\left.f L U C_{T C P 4}\right)$ sensors. Bars represent mean \pm SEM of three independent experiments. $n s$, non significant. 
Figure 6
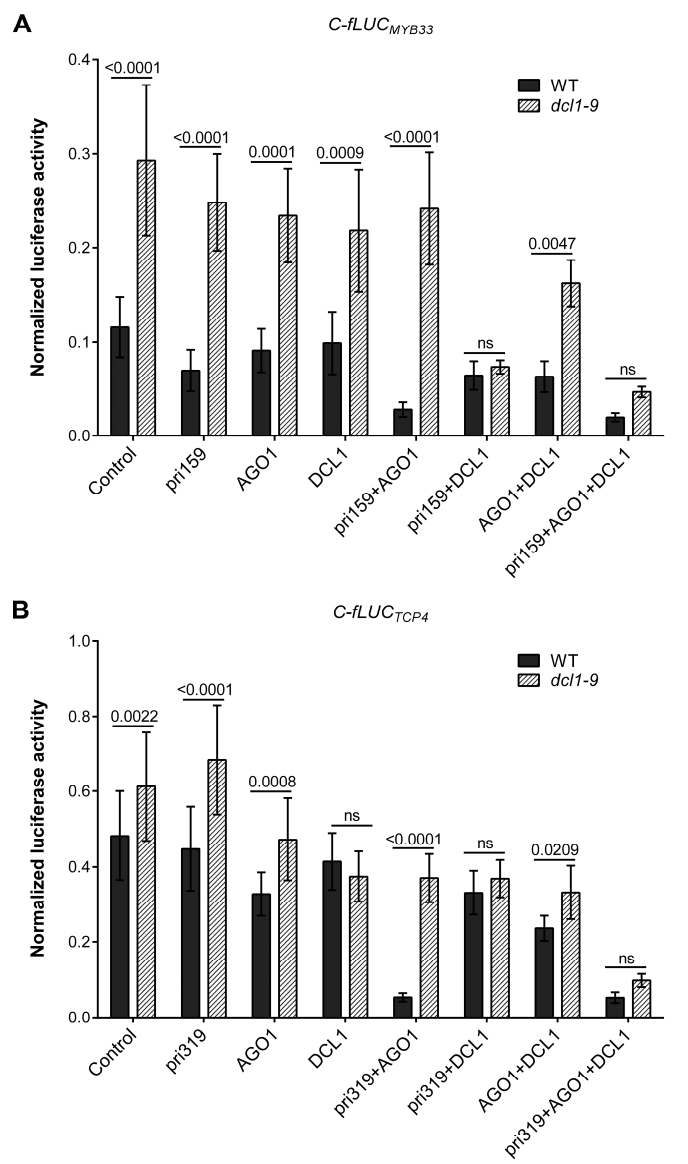

Figure 6. Use of miRNA sensors for dissection of miRNA pathways: monitoring miRNA activity in the dc/1-9 mutant. Graphs depict normalized luciferase activity of cleavable variants (C-fLUC) of sensors for miR159 (A, C-fLUC $C_{M Y B 33}$ ) and miR319 (B, C-fLUC $C_{T C P 4}$ ) activity in the dc/1-9 mutant and WT plants. $p$ values, two-way ANOVA and Fisher's LSD test. Bars represent mean \pm SEM of three independent experiments. $n s$, non significant. 


\section{Supplementary Data}

\section{Figure S1}
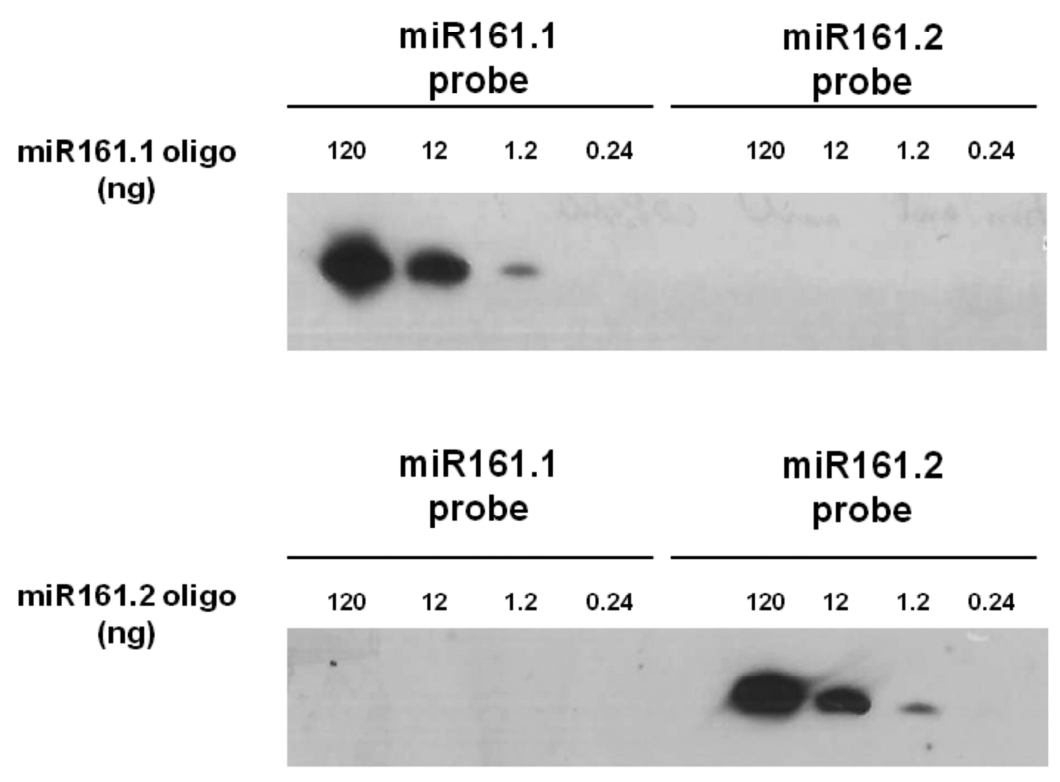

Figure S1. Specific probes distinguish between synthetic oligonucleotides corresponding to the sequences of miR161.1 and miR161.2. Small RNA blots show that probes for miR161.1 and miR161.2 are specific. Different amounts of oligonucleotides with the sequence of miR161.1 and miR161.2 were loaded in the top and bottom blots, respectively. 
Figure S2
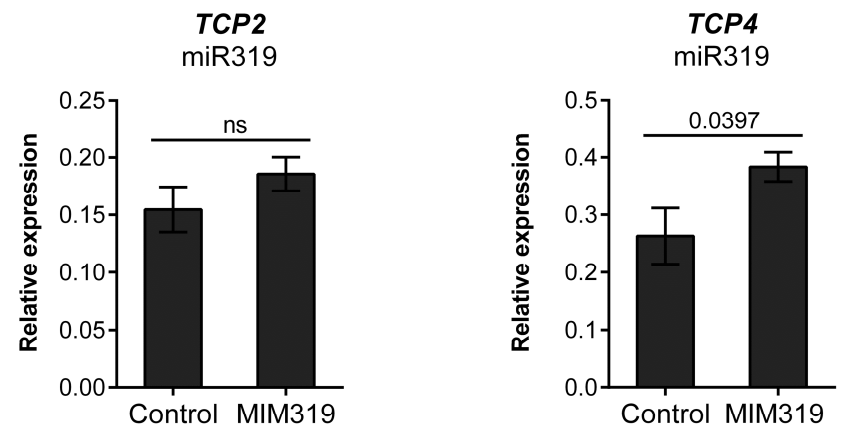

Figure S2. Relative quantification of TCP2 and TCP4 in stable plant lines overexpressing a miR319 target mimic (MIM319). Overexpression of miR319 target mimic (MIM319) in stable plant lines and protoplasts (Figure $2 \mathrm{~B}$ ) is comparable. Relative quantification by qRT-PCR. EIF4 was used as reference gene for normalization. The graphs represent mean $\pm S E M$ of three biological replicates. $p$ values, ratio paired t test. 
Figure S3
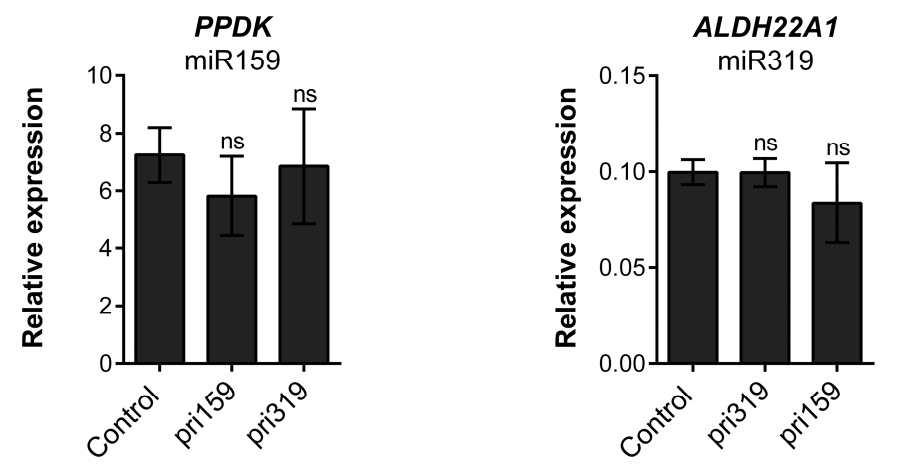

Figure S3. Overexpression of primary miRNAs 159 a and 319a does not affect PPDK and $A L D H 22$ levels. Relative quantification by qRT-PCR. The graphs represent mean $\pm S E M$ of at least three independent biological replicates. ACT2 was used as a reference gene. Numbers refer to $p$ values, ratio paired $t$-test comparing each primary miRNA to a control plasmid. 
Figure S4
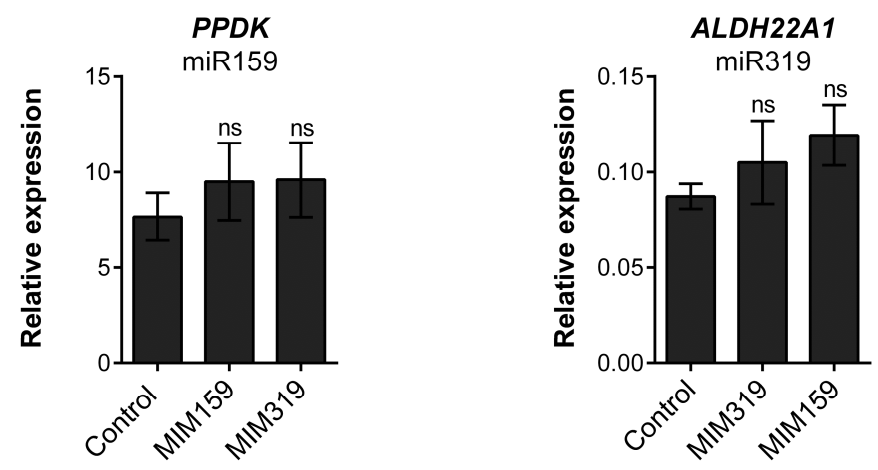

Figure S4. Overexpression of target mimics for miR159 (MIM159) and miR319 (MIM319) does not affect PPDK and $A L D H 22$ levels. Relative quantification by qRT-PCR. The graphs represent mean $\pm S E M$ of at least three independent biological replicates. ACT2 was used as a reference gene. Numbers refer to $p$ values, ratio paired $t$-test comparing each target mimic to a control plasmid. 


\section{Figure S5}

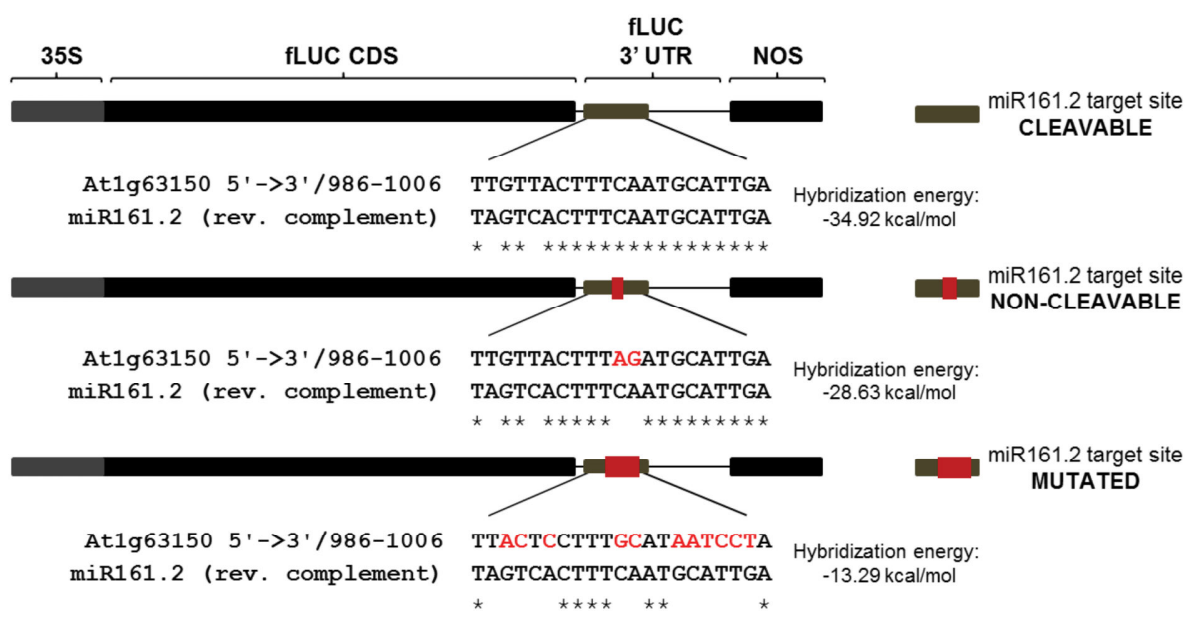

Figure S5. Firefly luciferase ( $f L U C$ ) miRNA sensor to monitor miRNA 161.2 activity in Arabidopsis protoplasts. The At1g63150 target site for miR161.2 was introduced in the 3'UTR of $f L U C$, generating the corresponding the cleavable reporter. As negative controls, we used a non-cleavable sensor variant with mutations in positions corresponding to nucleotides 10 and 11 of miR161.2 or a mutated sensor variant with a higher number of mutations. Mutated nucleotides are shown in red. In all cases, expression of the the fLUC coding sequence (CDS) is under the control of the $35 S$ promoter and the NOS terminator. 


\section{Figure S6}

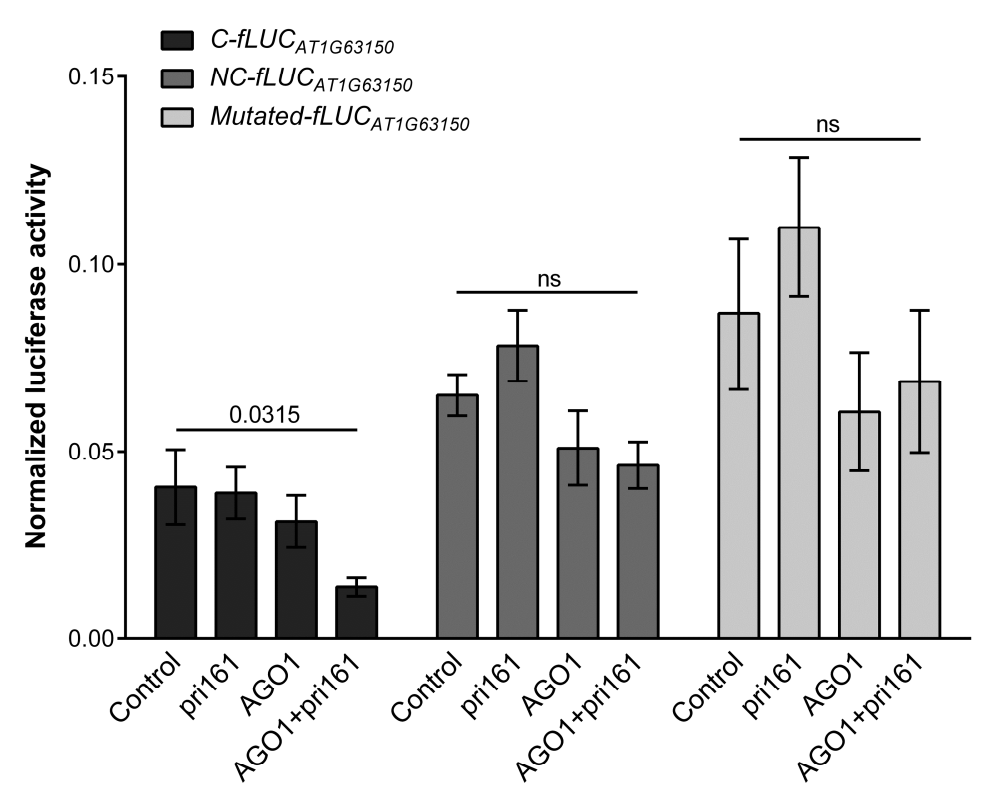

Figure S6. Non-cleavable miRNA sensor for miR161.2 behaves essentially as a variant with higher number of mutations. Graph depicts normalized luciferase activity of cleavable variants $(C-f L U C)$, non-cleavable $(N C$ - $f L U C)$ and mutated (Mutated-fLUC) versions of the sensor for miR161.2 activity. Bars represent mean \pm SEM of two independent experiments. $p$ values, ratio paired $t$ test. 


\section{Figure S7}

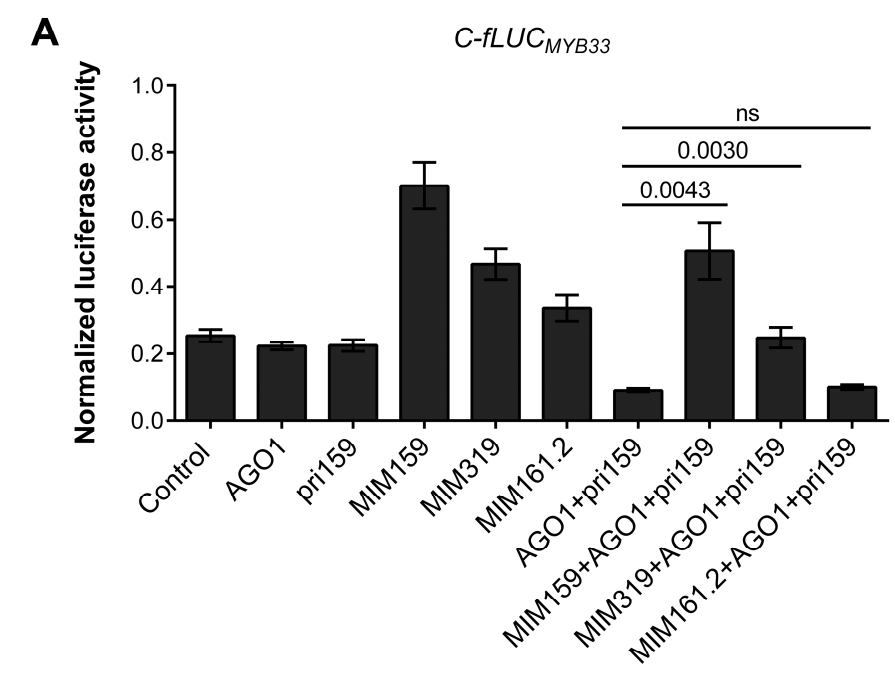

B

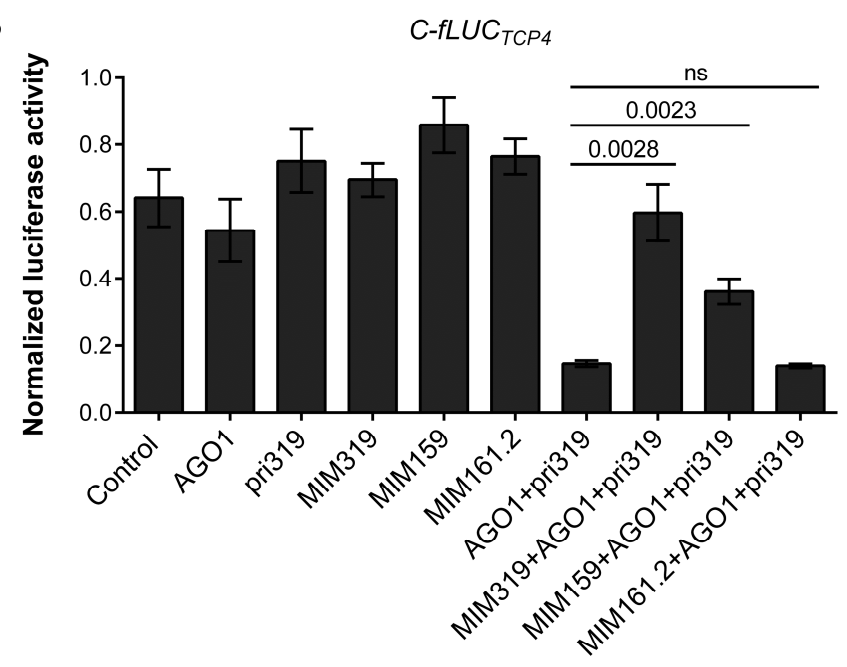

C

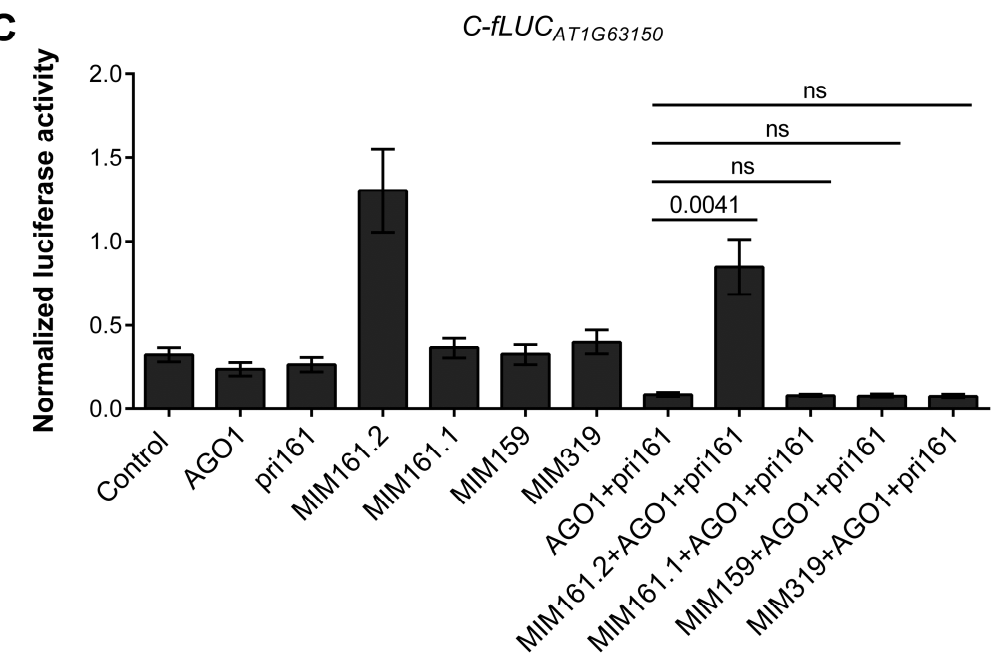

Figure S7. Luciferase-based miRNA sensors are highly specific tools to measure miRNA activity in Arabidopsis protoplasts. Graphs depict normalized luciferase activity of cleavable variants $(C-f L U C)$ of sensors for miR159 (A, fLUC $\left.C_{M Y B 33}\right)$, miR319 (B, fLUC TCP4) and miR161.2 (C), $f L U C_{A T 1663150}$ ) activity. Bars represent mean \pm SEM of three independent experiments. $p$ values obtained by one-way ANOVA and Fisher's LSD test. 


\section{Figure S8}
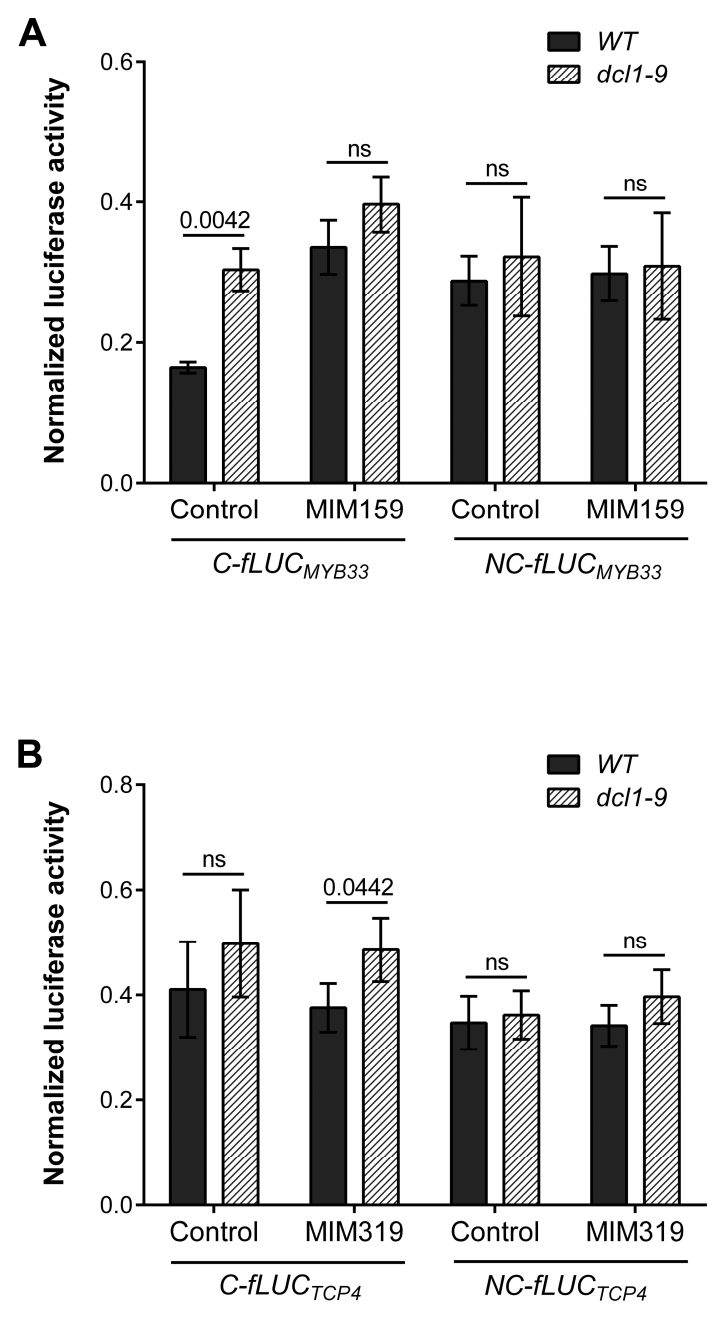

Figure S8. Basal activities of cleavable and non-cleavable reporters in WT and dc/1-9. Graphs depict normalized luciferase activity of cleavable (C-fLUC) and non-cleavable (NCfLUC) variants of sensors for miR159 (A, $\left.f L U C_{M Y B 33}\right)$ and miR319 (B, $\left.f L U C_{T C P 4}\right)$ activity. Bars represent mean \pm SEM of three independent experiments. $p$ values, two-way ANOVA and Fisher's LSD test. 


\section{Figure S9}

A $C-f L U C_{M Y B 33}$

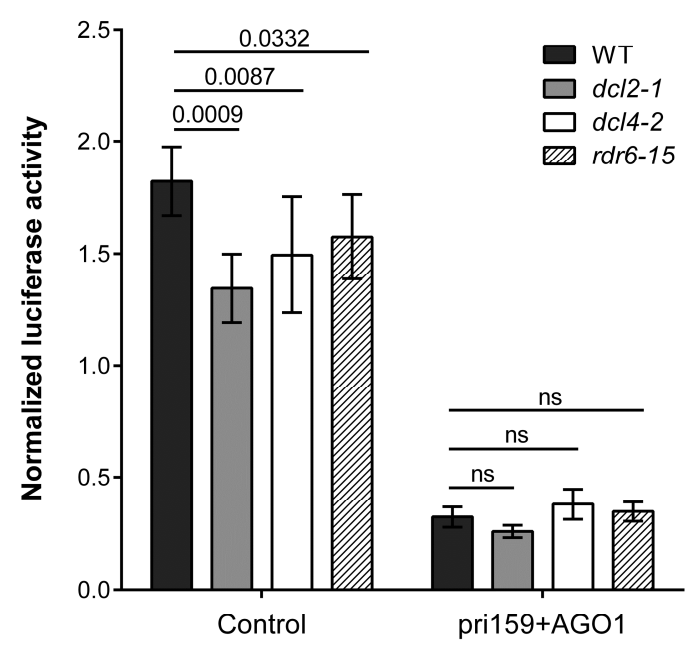

B

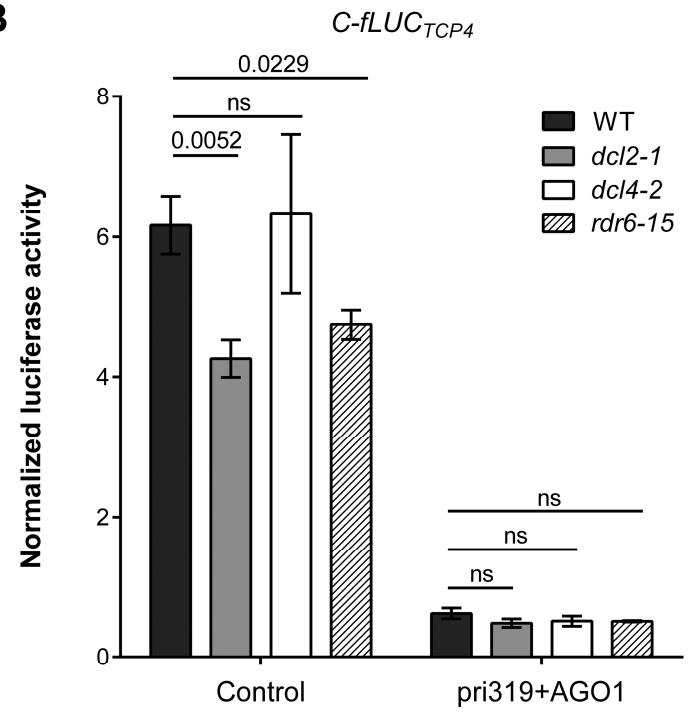

Figure S9. Repression of miRNA reporters does not require the formation of secondary siRNAs. Graphs depict normalized luciferase activity of cleavable (C-fLUC) sensors for miR159 (A, $\left.f L U C_{M Y B 33}\right)$ and miR319 (B, $\left.f L U C_{T C P 4}\right)$ activity in $d c / 2-1, d c / 4-2, r d r 6-15$ and WT protoplasts. Bars represent mean \pm SEM of two independent experiments. $p$ values, two-way ANOVA and Fisher's LSD test. 


\begin{tabular}{|c|c|c|c|c|}
\hline \multicolumn{5}{|c|}{ SUPPLEMENTARY TABLE 1. LIST OF ALL THE PRIMERS USED. } \\
\hline \multicolumn{5}{|l|}{ Cloning primers } \\
\hline Primer name & Sequence $\left(5^{\prime}-3^{\prime}\right)$ & AGI & Gene & $\begin{array}{l}\text { Based on primers } \\
\text { from }\end{array}$ \\
\hline pri159a_Ncol_A & CATGCCAAAGGTCTTTACAGTTTGCTT & \multirow{2}{*}{ AT1G73687 } & \multirow{2}{*}{ pri159a } & \multirow[t]{2}{*}{ 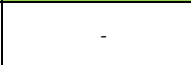 } \\
\hline pri159a_Pst_B & AAAACTGCAGAAGTCTCCAAAGAACCAAGGA & & & \\
\hline pri161_BamHI_A & CGGGATCCCTTCGTGTTATAAATTGTAAAC & \multirow{2}{*}{ AT1G48267 } & \multirow{2}{*}{ pri161 } & \multirow{2}{*}{-} \\
\hline pri161_Pstl_B & AAAACTGCAGCTTTCTCGCATCTGAAAAATAAC & & & \\
\hline pri319a_BamHI_A & GCGGATCCTCGAGCAAACACACGCTCGGACGCATA & \multirow{2}{*}{ AT4G23713 } & \multirow{2}{*}{ pri319a } & \multirow{2}{*}{ Schwab et al., 2006} \\
\hline pri319a_Pstl_B & TGCACTGCAGACTAGTCATGGCGATGCCTTAAATAAAGATA & & & \\
\hline IPS1_BamHI_A & CGGGATCCAAACACCACAAAAACAAAAG & \multirow{2}{*}{ At3g09922 } & \multirow{2}{*}{ IPS1 } & \multirow{2}{*}{-} \\
\hline IPS1_Pstl_B & AAAACTGCAGAAGAGGAATTCACTATAAAG & & & \\
\hline Mutagenesis primers & & & & \\
\hline Primer name & Sequence $\left(5^{\prime}-3^{\prime}\right)$ & & & $\begin{array}{l}\text { Based on primers } \\
\text { from } \\
\end{array}$ \\
\hline LUC_UTR_TCP4_A & AAGTCCAAATTGTAAAATAGGGGTCCCCTTCAGTCCAGGTAACTGTATTCAGCGAT & & & \\
\hline LUC_UTR_TCP4_B & ATCGCTGAATACAGTTACCTGGACTGAAGGGGACCCCTATTTTACAATTTGGACTT & & & - \\
\hline LUC_UTR_NC_TCP4_A & AAGTCCAAATTGTAAAATAGGGGTCCCAGTCAGTCCAGGTAACTGTATTCAGCGAT & & & \\
\hline LUC_UTR_NC_TCP4_B & ATCGCTGAATACAGTTACCTGGACTGACTGGGACCCCTATTTTACAATTTGGACTT & & & - \\
\hline LUC_UTR_MYB33_A & AAGTCCAAATTGTAAAATTGGAGCTCCCTTCATTCCAATGTAACTGTATTCAGCGAT & & & 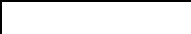 \\
\hline LUC_UTR_MYB33_B & ATCGCTGAATACAGTTACATTGGAATGAAGGGAGCTCCAATTTTACAATTTGGACTT & & & - \\
\hline LUC_UTR_NC_MYB33_A & AAGTCCAAATTGTAAAATTGGAGCTCCCGACATTCCAATGTAACTGTATTCAGCGAT & & & \\
\hline LUC_UTR_NC_MYB33_B & ATCGCTGAATACAGTTACATTGGAATGTCGGGAGCTCCAATTTTACAATTTGGACTT & & & - \\
\hline LUC_UTR_AT1G63150_A & CGGAAAGTCCAAATTGTAAAATTTGTTACTTTCAATGCATTGAGTAACTGTATTCAGCGATGACG & & & - \\
\hline LUC_UTR_AT1G63150_B & CGTCATCGCTGAATACAGTTACTCAATGCATTGAAAGTAACAAATTTTACAATTTGGACTTTCCG & & & - \\
\hline LUC_UTR_NC_AT1G63150_A & TGTAAAATTTGTTACTTTAGATGCATTGAGTAACTGTA & & & - \\
\hline LUC_UTR_NC_AT1G63150_B & TACAGTTACTCAATGCATCTAAAGTAACAAATTTTACA & & & - \\
\hline LUC_UTR_MUT_AT1G63150_A & CGGAAAGTCCAAATTGTAAAATTTACTCCTTTGCATAATCCTAGTAACTGTATTCAGCGATGACG & & & - \\
\hline LUC_UTR_MUT_AT1G63150_B & CGTCATCGCTGAATACAGTTACTAGGATTATGCAAAGGAGTAAATTTTACAATTTGGACTTTCCG & & & - \\
\hline AG01_D760A_A & TTATATTTGGTGCTGCTGTTACCCACCCTCA & & & \\
\hline AG01_D760A_B & TGAGGGTGGGTAACAGCAGCACCAAATATAA & & & - \\
\hline AG01_D848A_A & CATCTTCTACAGGGCTGGAGTCAGTGAGGG & & & \\
\hline AG01_D848A_B & CCCTCACTGACTCCAGCCCTGTAGAAGATG & & & - \\
\hline IPS1_MIM159_A & TTCCGAGGGGAACCGAAGCTTTTGGATTGATTGAGGGAGCTCTTTTTCTAGAGGGAGATAAACA & & & Todesco et al. 2010 \\
\hline IPS1_MIM159_B & TGTTTATCTCCCTCTAGAAAAAGAGCTCCCTCAATCAATCCAAAAGCTTCGGTTCCCCTCGGAA & & & Todesco et al., 2010 \\
\hline IPS1_MIM319_A & TTCCGAGGGGAACCGAAGCTTTGGACTGAATAGAGGAGCTCCTTTTCTAGAGGGAGATAAACA & & & Todesco et al. 2010 \\
\hline IPS1_MIM319_B & TGTTIATCTCCCTCTAGAAAAGGAGCTCCTCTATTCAGTCCAAAGCTTCGGTTCCCCTCGGAA & & & Iodesco et al., 2010 \\
\hline qRT-PCR primers & & & & \\
\hline Primer name & Sequence $\left(5^{\prime}-3^{\prime}\right)$ & $\mathrm{AGI}$ & Gene & Described in \\
\hline TCP2_A & СTCTGTTTCTTCTGCTTGTGGT & AT4G18390 & $T C P 2$ & Confraria et al 2013 \\
\hline TCP2_B & GCTTCGTATAAGCTTTGTCTGC & & ICP2 & Coniraria et al., 2013 \\
\hline TCP4_A & ACGTCGTTTCAGCCAGTTCT & AT3G15030 & TCP4 & Confraria et al 2013 \\
\hline TCP4_B & TGGAGATGGATTGGTGATGA & Al3015030 & & \\
\hline MYB33_A & CTACGGATGGCATTGTTCCT & AT5G06100 & MYB33 & - \\
\hline MYB33_B & TGACGATTTCTTCCACTGGTC & A15000100 & MITDJ3 & - \\
\hline PPDK_A & AGCCTCAAGGTTGGGATATG & At49 15530 & PPDK & - \\
\hline PPDK_B & TGGAACCCTGAAAGGAGAAC & Al4g13530 & & \\
\hline ALDH22_A & CATGTTGCACAAGTGGCTGT & AT3G66658 & $A L P H 22 A 1$ & - \\
\hline ALDH22_B & CTTGGCCAATGAATGCAGTA & Al3666658 & ALUHLZAI & - \\
\hline ACT2_A & GGCAAGTCATCACGATTGG & $A+3918780$ & $A C T 2$ & Confrario ot at 2013 \\
\hline ACT2_B & CTTCCATTCCCACAAACGAG & Atsg $18 / 80$ & 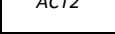 & Conirarta et al., 2013 \\
\hline EIF4_A & TCATAGATCTGGTCCTTAAACC & At3013920 & EIF4 & Baena-González et \\
\hline EIF4_B & GGCAGTCTCTTCGTGCTGAC & Alsglog20 & 21154 & al., 2007 \\
\hline LUC_A & GGAAAACTCGACGCAAGAAA & & LUC & - \\
\hline LUC_B & TCGTCATCGCTGAATACAGTT & & LUC & - \\
\hline$\beta$-GUS_A & ACAGCCAAAAGCCAGACAGA & & B-GUS & - \\
\hline$\beta$-GUS_B & TGACGACCAAAGCCAGTAAA & & b-GUS & - \\
\hline Genotyping primers & & & & \\
\hline Primer name & Sequence $\left(5^{\prime}-3^{\prime}\right)$ & $\mathrm{AGI}$ & Gene & Described in \\
\hline dcl1-9_TDNA & GATGCACTCGAAATCAGCCAATTTTAGAC & & & \\
\hline$d c / 1-9+A$ & TCATCGACGGTGTTCAAGTTGGAG & At1g01040 & $D C L 1$ & Confraria et al., 2013 \\
\hline$d c / 1-9 \_B$ & TCCATCCTCTATCGCTCGTATTAAC & & & \\
\hline Probes for small RNA blots & & & & \\
\hline Probe name & Sequence $\left(5^{\prime}-3^{\prime}\right)$ & AGI & Gene & Described in \\
\hline U6 snRNA_NB & TCATCCTTGCGCAGGGGCCA & - & - & - \\
\hline miR159/miR319_NB & TAGAGCTCCCTTCAATCCAAA & - & - & - \\
\hline miR161.1_NB & CCCCGATGTAGTCACTTTCAA & - & - & - \\
\hline miR161.2_NB & TAGTCACTTTCAATGCATTGA & - & - & - \\
\hline
\end{tabular}




\begin{tabular}{|c|c|c|c|c|c|}
\hline \multicolumn{6}{|c|}{ SUPPLEMENTARY TABLE 2. LIST OF ALL THE CONSTRUCTS USED. } \\
\hline Name & Vector & Insert & Resistance & Brief description & Previously described \\
\hline & pHBT95 & mER7 & Ampicillin & Control DNA & Kovtun et al., 1998 \\
\hline & pHBT95 & pri319a & Ampicillin & Genomic sequence for primary miR319a & Gift from Guillaume Tena \\
\hline pCM27 & pHBT95 & pri159a & Ampicillin & Genomic sequence for primary miR159a & - \\
\hline pCM26 & pHBT95 & pri161 & Ampicillin & Genomic sequence for primary miR161 & - \\
\hline pAC26 & pHBT95 & MIM319 & Ampicillin & IPS1 containing target mimic for miR319 & - \\
\hline pAC24 & pHBT95 & MIM159 & Ampicillin & IPS1 containing target mimic for miR159 & - \\
\hline pAC18 & pHBT95 & MIM161.1 & Ampicillin & IPS1 containing target mimic for miR161.1 & - \\
\hline \multirow[t]{2}{*}{ pCM24 } & pHBT95 & MIM161.2 & Ampicillin & IPS1 containing target mimic for miR161.2 & - \\
\hline & pHBT95 & $\beta$-glucuronidase & Ampicillin & Transfection control & \begin{tabular}{|l|l} 
Baena-González et $a l$, , 2007 \\
\end{tabular} \\
\hline pCM33 & pUC18 & C-fLUC $C_{\text {MYB33 }}$ & Ampicillin & Target site for miR159 (from MYB33) introduced in 3'UTR of firefly luciferase & - \\
\hline pCM34 & pUC18 & NC-fLUC $C_{\text {MYB3 }}$ & Ampicillin & Non-cleavable target site for miR159 (mutated from MYB33) introduced in 3'UTR of firefly luciferase & - \\
\hline pCM30 & pUC18 & C-fLUC $\mathrm{CTP4}_{\mathrm{TTP}}$ & Ampicillin & Target site for miR319 (from TCP4) introduced in 3'UTR of firefly luciferase & - \\
\hline pCM31 & pUC18 & NC-fLUC TCP4 & Ampicillin & Non-cleavable target site for miR319 (mutated from TCP4) introduced in 3'UTR of firefly luciferase & - \\
\hline PEBGM3 & pUC18 & C-fLUC $C_{\text {AT1663150 }}$ & Ampicillin & Target site for miR161.2 (from AT1G63150) introduced in 3'UTR of firefly luciferase & - \\
\hline pAC22 & pUC18 & NC-fLUC $\mathrm{ATT}_{1663150}$ & Ampicillin & Non-cleavable target site for miR161.2 (mutated from AT1663150) introduced in 3'UTR of firefly luciferase & - \\
\hline pEBGM23 & pUC18 & Mutated-fLUC $C_{\text {A11663150 }}$ & Ampicillin & Mutated target site for miR161.2 (mutated from AT1G63150) introduced in 3'UTR of firefly luciferase & - \\
\hline PAE44 & p35S-HA-GW & AGO1 & Ampicillin & WT AGO1, HA tagged & - \\
\hline pPC64 & p35S-HA-GW & \begin{tabular}{|l|} 
AG01D760A \\
\end{tabular} & Ampicillin & Catalytic dead AGO1, HA tagged & - \\
\hline pPC65 & p35S-HA-GW & AG01D848A & Ampicillin & Catalytic dead AGO1, HA tagged & - \\
\hline pAE43 & p35S-HA-GW & \begin{tabular}{|l|} 
DCL1 \\
\end{tabular} & Ampicillin & WT DCLL, HA tagged & - \\
\hline
\end{tabular}




\section{Supplementary Data}

\section{Figure S1}
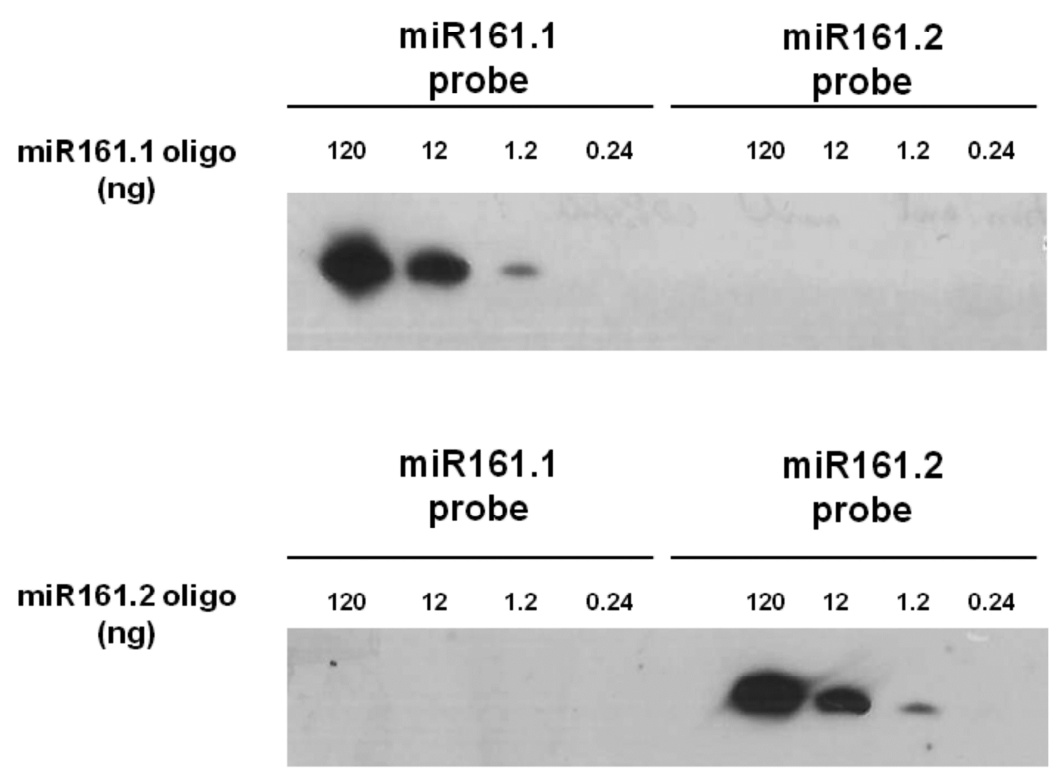

Figure S1. Specific probes distinguish between synthetic oligonucleotides corresponding to the sequences of miR161.1 and miR161.2. Small RNA blots show that probes for miR161.1 and miR161.2 are specific. Different amounts of oligonucleotides with the sequence of miR161.1 and miR161.2 were loaded in the top and bottom blots, respectively. 
Figure S2
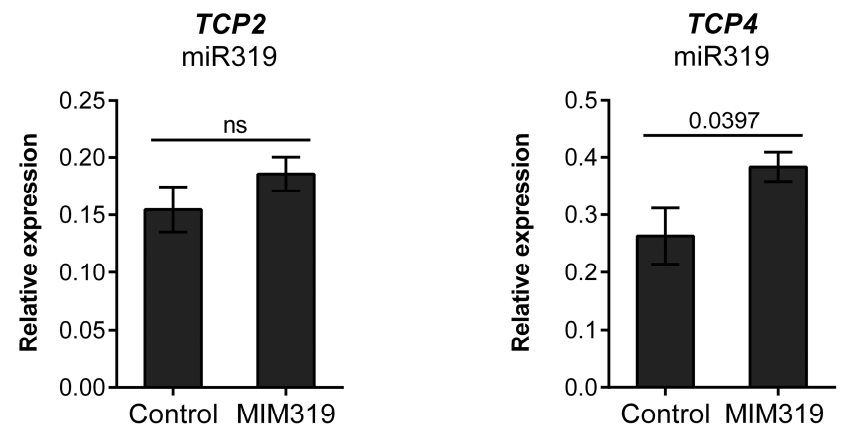

Figure S2. Relative quantification of TCP2 and TCP4 in stable plant lines overexpressing a miR319 target mimic (MIM319). Overexpression of miR319 target mimic (MIM319) in stable plant lines and protoplasts (Figure $2 \mathrm{~B}$ ) is comparable. Relative quantification by qRT-PCR. EIF4 was used as reference gene for normalization. The graphs represent mean $\pm S E M$ of three biological replicates. $p$ values, ratio paired t test. 
Figure S3
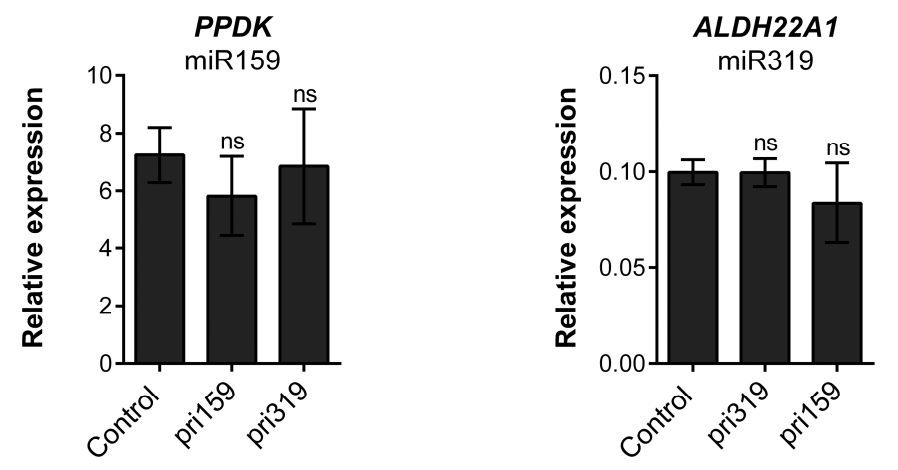

Figure S3. Overexpression of primary miRNAs 159 a and 319a does not affect PPDK and $A L D H 22$ levels. Relative quantification by qRT-PCR. The graphs represent mean $\pm S E M$ of at least three independent biological replicates. ACT2 was used as a reference gene. Numbers refer to $p$ values, ratio paired $t$-test comparing each primary miRNA to a control plasmid. 
Figure S4
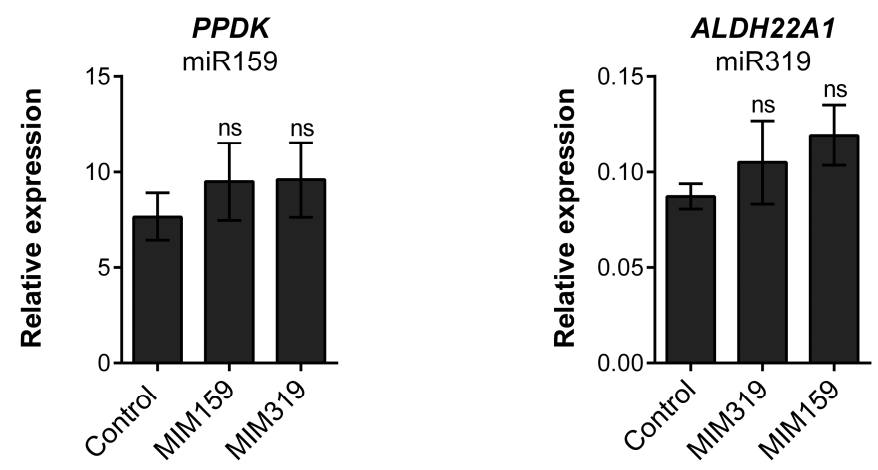

Figure S4. Overexpression of target mimics for miR159 (MIM159) and miR319 (MIM319) does not affect PPDK and $A L D H 22$ levels. Relative quantification by qRT-PCR. The graphs represent mean \pm SEM of at least three independent biological replicates. ACT2 was used as a reference gene. Numbers refer to $p$ values, ratio paired $t$-test comparing each target mimic to a control plasmid. 


\section{Figure S5}

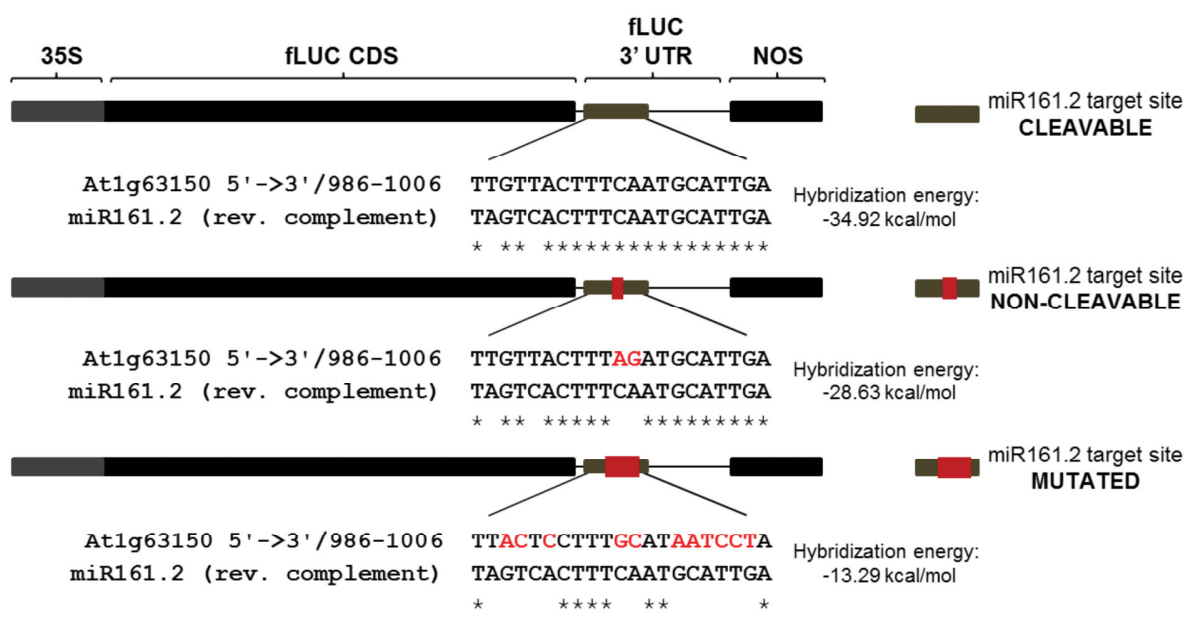

Figure S5. Firefly luciferase ( $f L U C$ ) miRNA sensor to monitor miRNA 161.2 activity in Arabidopsis protoplasts. The At1g63150 target site for miR161.2 was introduced in the 3'UTR of $f L U C$, generating the corresponding the cleavable reporter. As negative controls, we used a non-cleavable sensor variant with mutations in positions corresponding to nucleotides 10 and 11 of miR161.2 or a mutated sensor variant with a higher number of mutations. Mutated nucleotides are shown in red. In all cases, expression of the the fLUC coding sequence (CDS) is under the control of the $35 S$ promoter and the NOS terminator. 


\section{Figure S6}

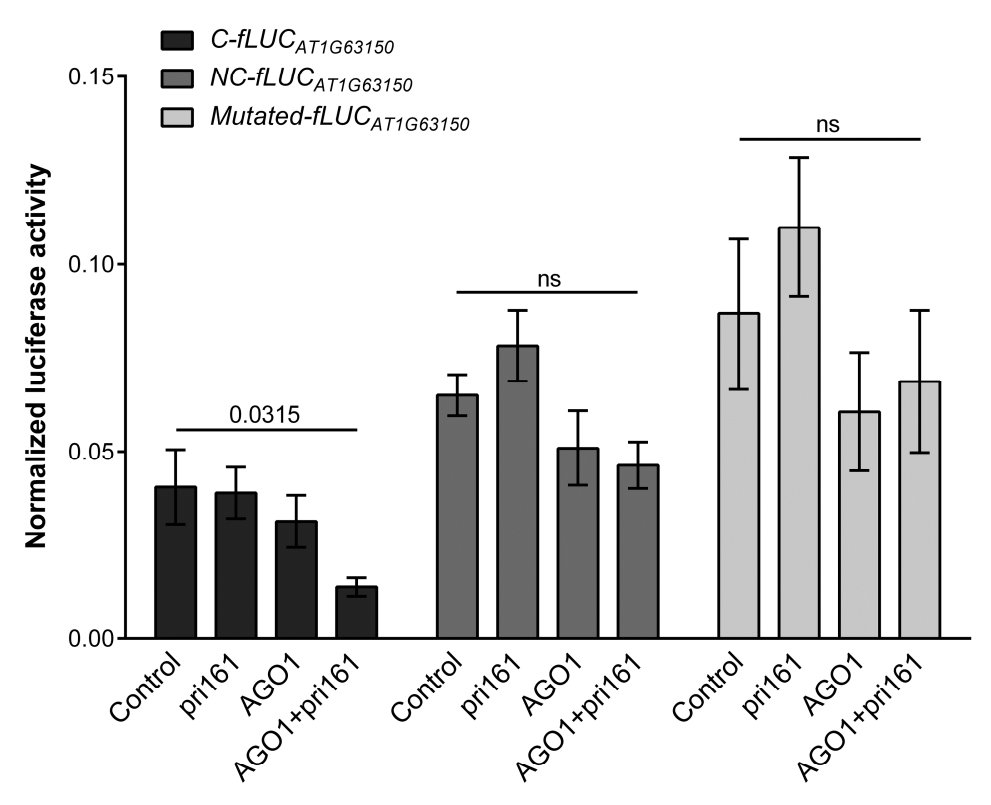

Figure S6. Non-cleavable miRNA sensor for miR161.2 behaves essentially as a variant with higher number of mutations. Graph depicts normalized luciferase activity of cleavable variants $(C-f L U C)$, non-cleavable $(N C$ - $f L U C)$ and mutated (Mutated-fLUC) versions of the sensor for miR161.2 activity. Bars represent mean \pm SEM of two independent experiments. $p$ values, ratio paired $t$ test. 


\section{Figure S7}

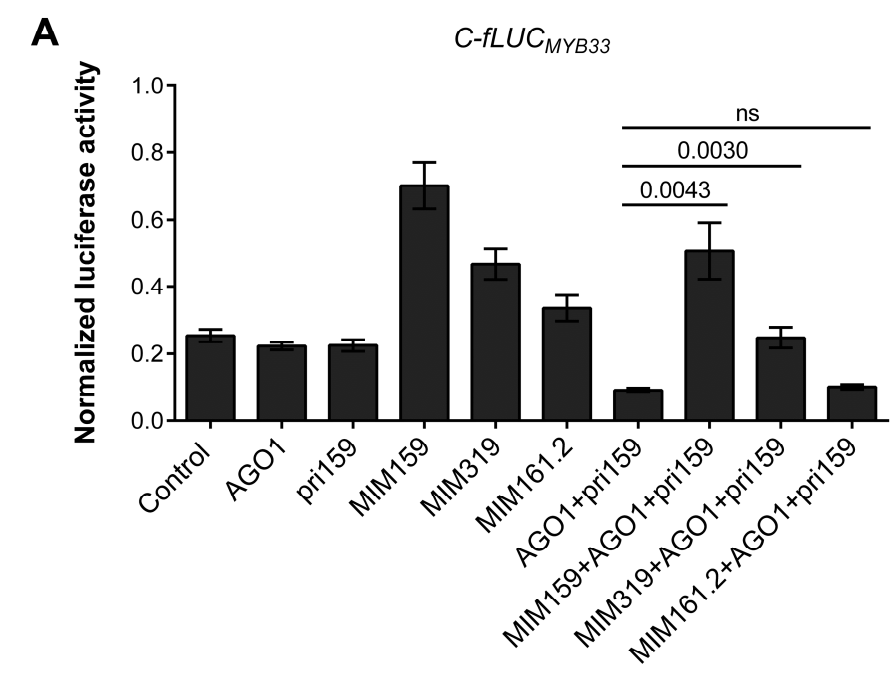

B

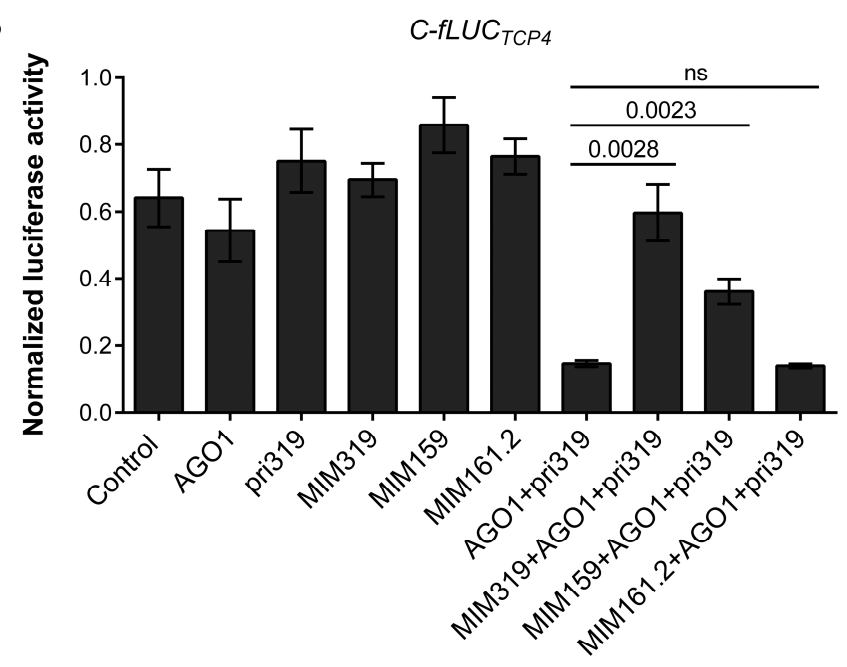

C

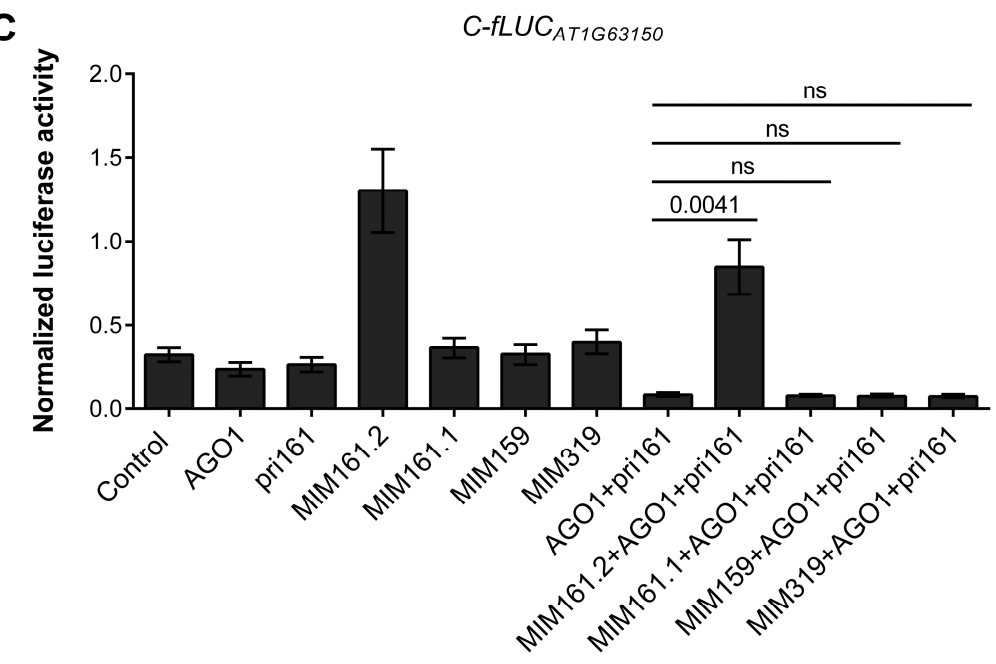

Figure S7. Luciferase-based miRNA sensors are highly specific tools to measure miRNA activity in Arabidopsis protoplasts. Graphs depict normalized luciferase activity of cleavable variants $(C-f L U C)$ of sensors for miR159 (A, fLUC $\left.C_{M Y B 33}\right)$, miR319 (B, fLUC TCP4) and miR161.2 (C), $f L U C_{A T 1663150}$ ) activity. Bars represent mean $\pm S E M$ of three independent experiments. $p$ values obtained by one-way ANOVA and Fisher's LSD test. 


\section{Figure S8}
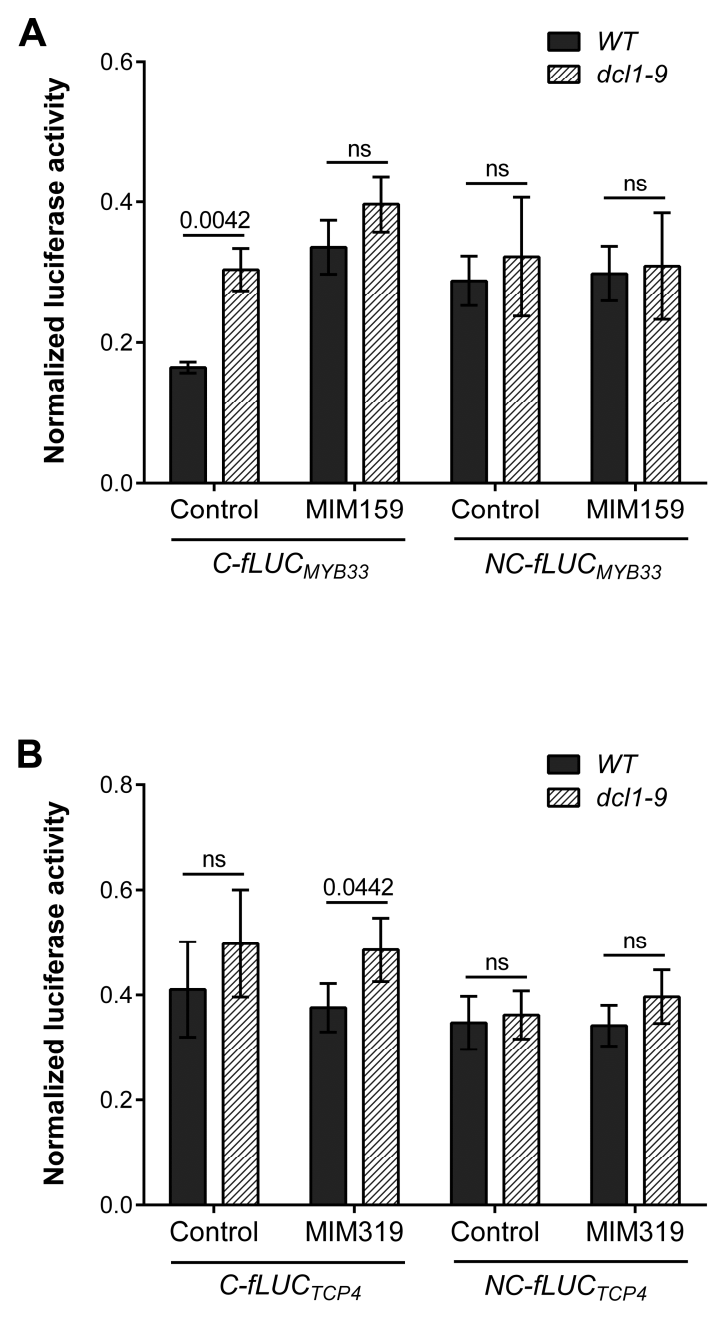

Figure S8. Basal activities of cleavable and non-cleavable reporters in WT and dc/1-9. Graphs depict normalized luciferase activity of cleavable (C-fLUC) and non-cleavable (NCfLUC) variants of sensors for miR159 (A, $\left.f L U C_{M Y B 33}\right)$ and miR319 (B, $\left.f L U C_{T C P 4}\right)$ activity. Bars represent mean \pm SEM of three independent experiments. $p$ values, two-way ANOVA and Fisher's LSD test. 


\section{Figure S9}

A $C-f L U C_{M Y B 33}$

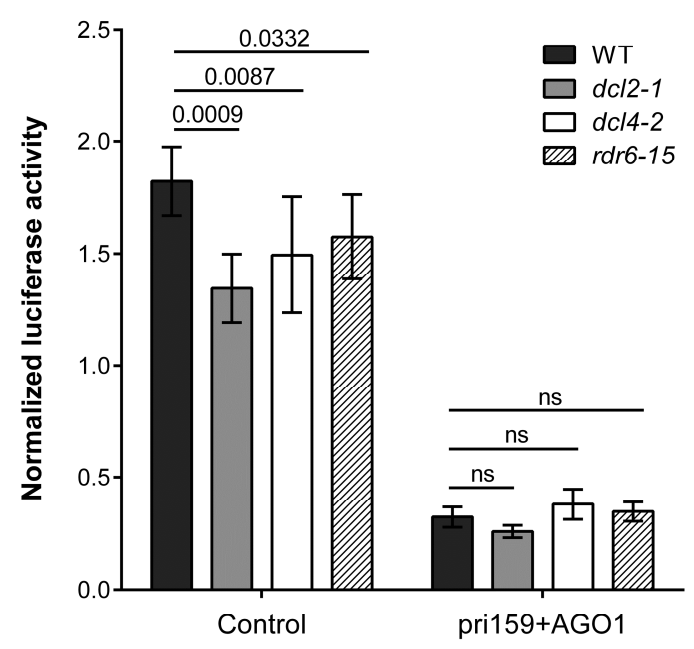

B

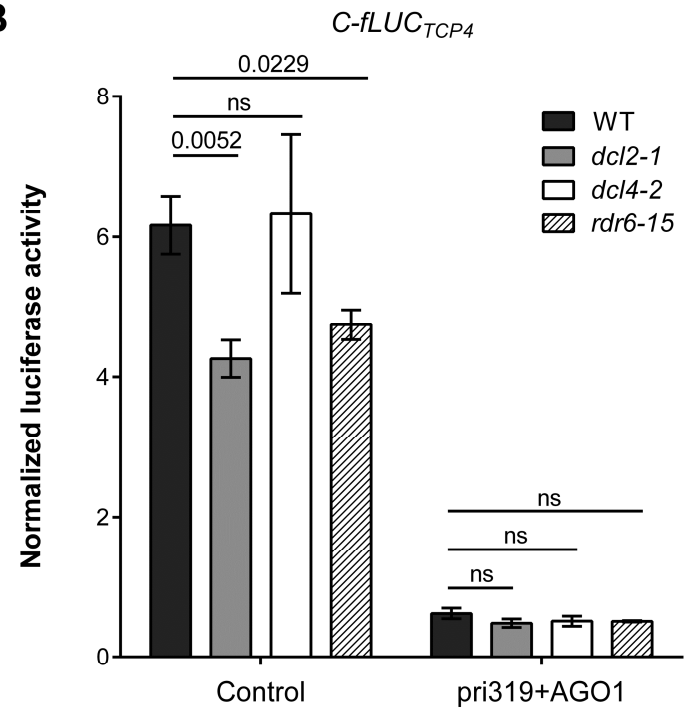

Figure S9. Repression of miRNA reporters does not require the formation of secondary siRNAs. Graphs depict normalized luciferase activity of cleavable (C-fLUC) sensors for miR159 (A, $\left.f L U C_{M Y B 33}\right)$ and miR319 (B, $\left.f L U C_{T C P 4}\right)$ activity in $d c / 2-1, d c / 4-2, r d r 6-15$ and WT protoplasts. Bars represent mean \pm SEM of two independent experiments. $p$ values, two-way ANOVA and Fisher's LSD test. 


\begin{tabular}{|c|c|c|c|c|}
\hline \multicolumn{5}{|c|}{ SUPPLEMENTARY TABLE 1. LIST OF ALL THE PRIMERS USED. } \\
\hline \multicolumn{5}{|l|}{ Cloning primers } \\
\hline Primer name & Sequence $\left(5^{\prime}-3^{\prime}\right)$ & AGI & Gene & $\begin{array}{l}\text { Based on primers } \\
\text { from }\end{array}$ \\
\hline pri159a_Ncol_A & CATGCCAAAGGTCTTTACAGTTTGCTT & \multirow{2}{*}{ AT1G73687 } & \multirow{2}{*}{ pri159a } & \multirow[t]{2}{*}{ 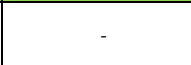 } \\
\hline pri159a_Pst_B & AAAACTGCAGAAGTCTCCAAAGAACCAAGGA & & & \\
\hline pri161_BamHI_A & CGGGATCCCTTCGTGTTATAAATTGTAAAC & \multirow{2}{*}{ AT1G48267 } & \multirow{2}{*}{ pri161 } & \multirow{2}{*}{-} \\
\hline pri161_Pstl_B & AAAACTGCAGCTTTCTCGCATCTGAAAAATAAC & & & \\
\hline pri319a_BamHI_A & GCGGATCCTCGAGCAAACACACGCTCGGACGCATA & \multirow{2}{*}{ AT4G23713 } & \multirow{2}{*}{ pri319a } & \multirow{2}{*}{ Schwab et al., 2006} \\
\hline pri319a_Pstl_B & TGCACTGCAGACTAGTCATGGCGATGCCTTAAATAAAGATA & & & \\
\hline IPS1_BamHI_A & CGGGATCCAAACACCACAAAAACAAAAG & \multirow{2}{*}{ At3g09922 } & \multirow{2}{*}{ IPS1 } & \multirow{2}{*}{-} \\
\hline IPS1_Pstl_B & AAAACTGCAGAAGAGGAATTCACTATAAAG & & & \\
\hline Mutagenesis primers & & & & \\
\hline Primer name & Sequence $\left(5^{\prime}-3^{\prime}\right)$ & & & $\begin{array}{l}\text { Based on primers } \\
\text { from } \\
\end{array}$ \\
\hline LUC_UTR_TCP4_A & AAGTCCAAATTGTAAAATAGGGGTCCCCTTCAGTCCAGGTAACTGTATTCAGCGAT & & & \\
\hline LUC_UTR_TCP4_B & ATCGCTGAATACAGTTACCTGGACTGAAGGGGACCCCTATTTTACAATTTGGACTT & & & - \\
\hline LUC_UTR_NC_TCP4_A & AAGTCCAAATTGTAAAATAGGGGTCCCAGTCAGTCCAGGTAACTGTATTCAGCGAT & & & \\
\hline LUC_UTR_NC_TCP4_B & ATCGCTGAATACAGTTACCTGGACTGACTGGGACCCCTATTTTACAATTTGGACTT & & & - \\
\hline LUC_UTR_MYB33_A & AAGTCCAAATTGTAAAATTGGAGCTCCCTTCATTCCAATGTAACTGTATTCAGCGAT & & & 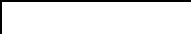 \\
\hline LUC_UTR_MYB33_B & ATCGCTGAATACAGTTACATTGGAATGAAGGGAGCTCCAATTTTACAATTTGGACTT & & & - \\
\hline LUC_UTR_NC_MYB33_A & AAGTCCAAATTGTAAAATTGGAGCTCCCGACATTCCAATGTAACTGTATTCAGCGAT & & & \\
\hline LUC_UTR_NC_MYB33_B & ATCGCTGAATACAGTTACATTGGAATGTCGGGAGCTCCAATTTTACAATTTGGACTT & & & - \\
\hline LUC_UTR_AT1G63150_A & CGGAAAGTCCAAATTGTAAAATTTGTTACTTTCAATGCATTGAGTAACTGTATTCAGCGATGACG & & & - \\
\hline LUC_UTR_AT1G63150_B & CGTCATCGCTGAATACAGTTACTCAATGCATTGAAAGTAACAAATTTTACAATTTGGACTTTCCG & & & - \\
\hline LUC_UTR_NC_AT1G63150_A & TGTAAAATTTGTTACTTTAGATGCATTGAGTAACTGTA & & & - \\
\hline LUC_UTR_NC_AT1G63150_B & TACAGTTACTCAATGCATCTAAAGTAACAAATTTTACA & & & - \\
\hline LUC_UTR_MUT_AT1G63150_A & CGGAAAGTCCAAATTGTAAAATTTACTCCTTTGCATAATCCTAGTAACTGTATTCAGCGATGACG & & & - \\
\hline LUC_UTR_MUT_AT1G63150_B & CGTCATCGCTGAATACAGTTACTAGGATTATGCAAAGGAGTAAATTTTACAATTTGGACTTTCCG & & & - \\
\hline AG01_D760A_A & TTATATTTGGTGCTGCTGTTACCCACCCTCA & & & \\
\hline AG01_D760A_B & TGAGGGTGGGTAACAGCAGCACCAAATATAA & & & - \\
\hline AG01_D848A_A & CATCTTCTACAGGGCTGGAGTCAGTGAGGG & & & \\
\hline AG01_D848A_B & CCCTCACTGACTCCAGCCCTGTAGAAGATG & & & - \\
\hline IPS1_MIM159_A & TTCCGAGGGGAACCGAAGCTTTTGGATTGATTGAGGGAGCTCTTTTTCTAGAGGGAGATAAACA & & & Todesco et al. 2010 \\
\hline IPS1_MIM159_B & TGTTTATCTCCCTCTAGAAAAAGAGCTCCCTCAATCAATCCAAAAGCTTCGGTTCCCCTCGGAA & & & Todesco et al., 2010 \\
\hline IPS1_MIM319_A & TTCCGAGGGGAACCGAAGCTTTGGACTGAATAGAGGAGCTCCTTTTCTAGAGGGAGATAAACA & & & Todesco et al. 2010 \\
\hline IPS1_MIM319_B & TGTTIATCTCCCTCTAGAAAAGGAGCTCCTCTATTCAGTCCAAAGCTTCGGTTCCCCTCGGAA & & & Iodesco et al., 2010 \\
\hline qRT-PCR primers & & & & \\
\hline Primer name & Sequence $\left(5^{\prime}-3^{\prime}\right)$ & $\mathrm{AGI}$ & Gene & Described in \\
\hline TCP2_A & СTCTGTTTCTTCTGCTTGTGGT & AT4G18390 & $T C P 2$ & Confraria et al 2013 \\
\hline TCP2_B & GCTTCGTATAAGCTTTGTCTGC & & ICP2 & Coniraria et al., 2013 \\
\hline TCP4_A & ACGTCGTTTCAGCCAGTTCT & AT3G15030 & TCP4 & Confraria et al 2013 \\
\hline TCP4_B & TGGAGATGGATTGGTGATGA & Al3015030 & & \\
\hline MYB33_A & CTACGGATGGCATTGTTCCT & AT5G06100 & MYB33 & - \\
\hline MYB33_B & TGACGATTTCTTCCACTGGTC & A15000100 & MITDJ3 & - \\
\hline PPDK_A & AGCCTCAAGGTTGGGATATG & At49 15530 & PPDK & - \\
\hline PPDK_B & TGGAACCCTGAAAGGAGAAC & Al4g13530 & & \\
\hline ALDH22_A & CATGTTGCACAAGTGGCTGT & AT3G66658 & $A L P H 22 A 1$ & - \\
\hline ALDH22_B & CTTGGCCAATGAATGCAGTA & Al3666658 & ALUHLZAI & - \\
\hline ACT2_A & GGCAAGTCATCACGATTGG & $A+3918780$ & $A C T 2$ & Confrario ot at 2013 \\
\hline ACT2_B & CTTCCATTCCCACAAACGAG & Atsg $18 / 80$ & 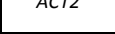 & Conirarta et al., 2013 \\
\hline EIF4_A & TCATAGATCTGGTCCTTAAACC & At3013920 & EIF4 & Baena-González et \\
\hline EIF4_B & GGCAGTCTCTTCGTGCTGAC & Alsglog20 & 21154 & al., 2007 \\
\hline LUC_A & GGAAAACTCGACGCAAGAAA & & LUC & - \\
\hline LUC_B & TCGTCATCGCTGAATACAGTT & & LUC & - \\
\hline$\beta$-GUS_A & ACAGCCAAAAGCCAGACAGA & & B-GUS & - \\
\hline$\beta$-GUS_B & TGACGACCAAAGCCAGTAAA & & b-GUS & - \\
\hline Genotyping primers & & & & \\
\hline Primer name & Sequence $\left(5^{\prime}-3^{\prime}\right)$ & $\mathrm{AGI}$ & Gene & Described in \\
\hline dcl1-9_TDNA & GATGCACTCGAAATCAGCCAATTTTAGAC & & & \\
\hline$d c / 1-9+A$ & TCATCGACGGTGTTCAAGTTGGAG & At1g01040 & $D C L 1$ & Confraria et al., 2013 \\
\hline$d c / 1-9 \_B$ & TCCATCCTCTATCGCTCGTATTAAC & & & \\
\hline Probes for small RNA blots & & & & \\
\hline Probe name & Sequence $\left(5^{\prime}-3^{\prime}\right)$ & AGI & Gene & Described in \\
\hline U6 snRNA_NB & TCATCCTTGCGCAGGGGCCA & - & - & - \\
\hline miR159/miR319_NB & TAGAGCTCCCTTCAATCCAAA & - & - & - \\
\hline miR161.1_NB & CCCCGATGTAGTCACTTTCAA & - & - & - \\
\hline miR161.2_NB & TAGTCACTTTCAATGCATTGA & - & - & - \\
\hline
\end{tabular}




\begin{tabular}{|c|c|c|c|c|c|}
\hline \multicolumn{6}{|c|}{ SUPPLEMENTARY TABLE 2. LIST OF ALL THE CONSTRUCTS USED. } \\
\hline Name & Vector & Insert & Resistance & Brief description & Previously described \\
\hline & pHBT95 & mER7 & Ampicillin & Control DNA & Kovtun et al., 1998 \\
\hline & pHBT95 & pri319a & Ampicillin & Genomic sequence for primary miR319a & Gift from Guillaume Tena \\
\hline pCM27 & pHBT95 & pri159a & Ampicillin & Genomic sequence for primary miR159a & - \\
\hline pCM26 & pHBT95 & pri161 & Ampicillin & Genomic sequence for primary miR161 & - \\
\hline pAC26 & pHBT95 & MIM319 & Ampicillin & IPS1 containing target mimic for miR319 & - \\
\hline pAC24 & pHBT95 & MIM159 & Ampicillin & IPS1 containing target mimic for miR159 & - \\
\hline pAC18 & pHBT95 & MIM161.1 & Ampicillin & IPS1 containing target mimic for miR161.1 & - \\
\hline \multirow[t]{2}{*}{ pCM24 } & pHBT95 & MIM161.2 & Ampicillin & IPS1 containing target mimic for miR161.2 & - \\
\hline & pHBT95 & $\beta$-glucuronidase & Ampicillin & Transfection control & \begin{tabular}{|l|l} 
Baena-González et $a l$, , 2007 \\
\end{tabular} \\
\hline pCM33 & pUC18 & C-fLUC $C_{\text {MYB33 }}$ & Ampicillin & Target site for miR159 (from MYB33) introduced in 3'UTR of firefly luciferase & - \\
\hline pCM34 & pUC18 & NC-fLUC $C_{\text {MYB3 }}$ & Ampicillin & Non-cleavable target site for miR159 (mutated from MYB33) introduced in 3'UTR of firefly luciferase & - \\
\hline pCM30 & pUC18 & C-fLUC $\mathrm{CTP4}_{\mathrm{TTP}}$ & Ampicillin & Target site for miR319 (from TCP4) introduced in 3'UTR of firefly luciferase & - \\
\hline pCM31 & pUC18 & NC-fLUC TCP4 & Ampicillin & Non-cleavable target site for miR319 (mutated from TCP4) introduced in 3'UTR of firefly luciferase & - \\
\hline PEBGM3 & pUC18 & C-fLUC $C_{\text {AT1663150 }}$ & Ampicillin & Target site for miR161.2 (from AT1G63150) introduced in 3'UTR of firefly luciferase & - \\
\hline pAC22 & pUC18 & NC-fLUC $\mathrm{ATT}_{1663150}$ & Ampicillin & Non-cleavable target site for miR161.2 (mutated from AT1663150) introduced in 3'UTR of firefly luciferase & - \\
\hline pEBGM23 & pUC18 & Mutated-fLUC $C_{\text {A11663150 }}$ & Ampicillin & Mutated target site for miR161.2 (mutated from AT1G63150) introduced in 3'UTR of firefly luciferase & - \\
\hline PAE44 & p35S-HA-GW & AGO1 & Ampicillin & WT AGO1, HA tagged & - \\
\hline pPC64 & p35S-HA-GW & \begin{tabular}{|l|} 
AG01D760A \\
\end{tabular} & Ampicillin & Catalytic dead AGO1, HA tagged & - \\
\hline pPC65 & p35S-HA-GW & AG01D848A & Ampicillin & Catalytic dead AGO1, HA tagged & - \\
\hline pAE43 & p35S-HA-GW & \begin{tabular}{|l|} 
DCL1 \\
\end{tabular} & Ampicillin & WT DCLL, HA tagged & - \\
\hline
\end{tabular}

